\title{
Iterative algorithms of common solutions for a hierarchical fixed point problem, a system of variational inequalities, and a split equilibrium problem in Hilbert spaces
}

\author{
Yali Zhao ${ }^{1 *} \mathbb{D}$, Xin Liu' and Ruonan Sun'
}

"Correspondence:

yalizhao2000@163.com

'School of Mathematical Science,

Bohai University, Liaoning, Jinzhou

121013, China

\section{黛 Springer}

\begin{abstract}
In this paper, we suggest and analyze an iterative algorithm to approximate a common solution of a hierarchical fixed point problem for nonexpansive mappings, a system of variational inequalities, and a split equilibrium problem in Hilbert spaces. Under some suitable conditions imposed on the sequences of parameters, we prove that the sequence generated by the proposed iterative method converges strongly to a common element of the solution set of these three kinds of problems. The results obtained here extend and improve the corresponding results of the relevant literature.
\end{abstract}

MSC: 49J30; 47H09; 47J20

Keywords: Hierarchical fixed point problem; Split equilibrium problem; System of variational inequalities; Iterative algorithm; Convergence

\section{Introduction}

Let $H_{1}$ and $H_{2}$ be two real Hilbert spaces, whose inner product and norm are denoted by $\langle\cdot, \cdot\rangle$ and $\|\cdot\|$. And let $C_{1}$ and $C_{2}$ be two nonempty closed convex subsets of $H_{1}$ and $H_{2}$, respectively. Recall that the mapping $T: C_{1} \rightarrow C_{1}$ is nonexpansive if $\|T x-T y\| \leq \| x-$ $y \|$ for all $x, y \in C_{1}$. We denote the fixed point set of $T$ by $\operatorname{Fix}(T)=\left\{x \in C_{1}: x=T x\right\}$. If $T$ is nonexpansive, then $\operatorname{Fix}(T)$ is nonempty, closed, and convex. Next, we consider the following three kinds of problems, which are paid attention to in our paper.

Problem 1 (Hierarchical fixed point problem (HFPP)) In 2006, Moudafi and Mainge [23] introduced and studied the following hierarchical fixed point problem (in short HFPP) for a nonexpansive mapping $T$ with respect to another nonexpansive mapping $S$ on $C_{1}$ : Find $x \in \operatorname{Fix}(T)$ such that

$$
\langle x-S x, y-x\rangle \geq 0, \quad \forall y \in \operatorname{Fix}(T)
$$

(c) The Author(s) 2021. This article is licensed under a Creative Commons Attribution 4.0 International License, which permits use, sharing, adaptation, distribution and reproduction in any medium or format, as long as you give appropriate credit to the original author(s) and the source, provide a link to the Creative Commons licence, and indicate if changes were made. The images or other third party material in this article are included in the article's Creative Commons licence, unless indicated otherwise in a credit line to the material. If material is not included in the article's Creative Commons licence and your intended use is not permitted by statutory regulation or exceeds the permitted use, you will need to obtain permission directly from the copyright holder. To view a copy of this licence, visit http://creativecommons.org/licenses/by/4.0/. 
which amounts to saying that $x \in \operatorname{Fix}(T)$ satisfies the variational inequality depending on a given criterion $S$, namely, find $x \in C_{1}$ such that

$$
0 \in(I-S) x+N_{\operatorname{Fix}(T)}(x)
$$

where $I$ is the identity mapping on $C_{1}$ and $N_{\mathrm{Fix}(T)}$ is the normal cone to $\operatorname{Fix}(T)$ at $x$ defined by

$$
N_{\mathrm{Fix}(T)}(x)= \begin{cases}\left\{u \in H_{1}:\langle y-x, u\rangle \leq 0, \forall y \in \operatorname{Fix}(T)\right\} & \text { if } x \in \operatorname{Fix}(T), \\ \varnothing & \text { otherwise }\end{cases}
$$

We know that the hierarchical fixed point problem links with some monotone variational inequalities and convex programming problems, see [39] and the references therein. In 2007, Moudafi [22] introduced the following Krasnoselski-Mann algorithm for solving $\operatorname{HFPP}(1)$ :

$$
x_{n+1}=\left(1-\alpha_{n}\right) x_{n}+\alpha_{n}\left(\sigma_{n} S x_{n}+\left(1-\sigma_{n}\right) T x_{n}\right) \text {, }
$$

where $\left\{\alpha_{n}\right\}$ and $\left\{\sigma_{n}\right\}$ are two real sequences in $(0,1)$.

On the other hand, in 2011, Ceng, Anasri, and Yao [8] proposed the following iterative method:

$$
x_{n+1}=P_{C}\left[\alpha_{n} \rho U\left(x_{n}\right)+\left(I-\alpha_{n} \mu F\right)\left(T\left(y_{n}\right)\right)\right] \text {, }
$$

where $U$ is a Lipschitzian mapping, and $F$ is a Lipschitzian and strongly monotone mapping. Under some approximate assumptions, they proved that the sequence $\left\{x_{n}\right\}$ generated by the above iterative algorithm converges strongly to the unique solution of the variational inequality

$$
\langle\rho U(x)-\mu F(x), y-x\rangle \geq 0, \quad \forall y \in \operatorname{Fix}(T) .
$$

Note that HFPP (2) is more general than HFPP (1).

Problem 2 (Split equilibrium problem (SEP)) Let $H$ be a real Hilbert space and $C$ be a nonempty closed convex subset of $H$. Let $F$ be a bifunction of $C \times C$ into $R$, where $R$ is the set of real numbers. The equilibrium problem(in short, EP) for $F: C \times C \rightarrow R$ is to find $x \in C$ such that

$$
F(x, y) \geq 0, \quad \forall y \in C,
$$

which was introduced and studied by Blum and Oettli [3]. It contains many problems, such as fixed point problem, variational inequality problem, Nash equilibrium problem, optimization problem, and complementarity problem as special cases, see, e.g., [1, 2, 20, 31] and the references therein. In 1997, Combettes and Hirstoaga [15] introduced an iterative scheme of finding the best approximation to the initial data when a set of solutions (3) is 
nonempty and proved a strong convergence theorem. We denote the solution set of EP (3) by $E P(F)=\{x \in C: F(x, y) \geq 0, \forall y \in C\}$.

Recently, Kazmi and Rizvi [21] considered the following split equilibrium problem (in short, SEP): Let $F_{1}: C_{1} \times C_{1} \rightarrow R$ and $F_{2}: C_{2} \times C_{2} \rightarrow R$ be two nonlinear bifunctions and $A: H_{1} \rightarrow H_{2}$ be a bounded linear operator, then the SEP is to find $x^{*} \in C_{1}$ such that

$$
F_{1}\left(x^{*}, x\right) \geq 0, \quad \forall x \in C_{1}
$$

and

$$
F_{2}\left(y^{*}, y\right) \geq 0, \quad \forall y \in C_{2},
$$

where $y^{*}=A x^{*} \in C_{2}$. The solution set of $\operatorname{SEP}(4)-(5)$ is denoted by $\Gamma=\left\{p \in E P\left(F_{1}\right): A p \in\right.$ $\left.E P\left(F_{2}\right)\right\}$. This formalism is also the core of modeling of many inverse problems arising in phase retrieval and other real word problems, for example, in sensor networks in computerized tomography, in intensity-modulated radiation therapy treatment planning, and data compression, see, e.g., $[5,6,12-14]$ and the references therein.

Problem 3 (System of variational inequalities (SVI)) Let $C_{1}$ be a nonempty closed convex subset of $H_{1}$ and $A, B: C_{1} \rightarrow H_{1}$ be two mappings. Ceng, Wang, and Yao [11] considered the following problem which finds $\left(x^{*}, y^{*}\right) \in C_{1} \times C_{1}$ such that

$$
\begin{cases}\left\langle\lambda_{1} A y^{*}+x^{*}-y^{*}, x-x^{*}\right\rangle \geq 0, & \forall x \in C_{1}, \\ \left\langle\lambda_{2} B x^{*}+y^{*}-x^{*}, x-y^{*}\right\rangle \geq 0, & \forall x \in C_{1} .\end{cases}
$$

Problem (6) is called a general system of variational inequalities, where $\lambda_{1}>0$ and $\lambda_{2}>0$ are constants. In 2015, Jitsupa et al. [19] introduced the following system of variational inequalities in a Hilbert space $H_{1}$, that is, finding $x_{i}^{*} \in C_{1}(i=1,2, \ldots, N)$ such that

$$
\begin{cases}\left\langle\lambda_{N} B_{N} x_{N}^{*}+x_{1}^{*}-x_{N}^{*}, x-x_{1}^{*}\right\rangle \geq 0, & \forall x \in C_{1}, \\ \left\langle\lambda_{N-1} B_{N-1} x_{N-1}^{*}+x_{N}^{*}-x_{N-1}^{*}, x-x_{N}^{*}\right\rangle \geq 0, & \forall x \in C_{1}, \\ \vdots & \\ \left\langle\lambda_{2} B_{2} x_{2}^{*}+x_{3}^{*}-x_{2}^{*}, x-x_{3}^{*}\right\rangle \geq 0, & \forall x \in C_{1}, \\ \left\langle\lambda_{1} B_{1} x_{1}^{*}+x_{2}^{*}-x_{1}^{*}, x-x_{2}^{*}\right\rangle \geq 0, & \forall x \in C_{1},\end{cases}
$$

which is called a more general system of variational inequalities, where $\lambda_{i}>0$ and $B_{i}: C_{1} \rightarrow$ $H_{1}$ is a nonlinear mapping for all $i \in\{1,2, \ldots, N\}$. The solution set of SVI (7) is denoted by $\operatorname{GSVI}\left(C_{1}, B_{i}\right)$.

In view of these different three kinds of problems, there are some new research results on numerical algorithm in the recent literature. Under the setting of uniformly convex Banach spaces, in [27-30], the Thakur three-step iterative process in the context of Suzukitype nonexpansive mappings or generalized nonexpansive mappings enriched with property (E) was studied, and a comparative numerical experiment was performed with the 
visualization of some convergence behaviors. In [25], an S-iteration technique for finding common fixed points for nonself quasi-nonexpansive mappings was developed, and convergence properties of the proposed algorithm were analyzed. And in [17], a hybrid projection algorithm for a countable family of mappings was considered, and the strong convergence of the algorithm converging to the common fixed point of the mappings was given. Very recently, Dadashi and Postolache [18] constructed a forward-backward splitting algorithm for approximating a zero of the sum of an $\alpha$-inverse strongly monotone operator and a maximal monotone operator. They proved the strong convergence theorem under mild conditions. Especially, they added a nonexpansive mapping in the algorithm and proved that the generated sequence converged strongly to a common element of the fixed point set of a nonexpansive mapping and the zero point set of the sum of monotone operators. They also applied their main result both to equilibrium problems and convex programming.

On the other hand, Ceng et al. [9] introduced a hybrid viscosity extragradient method for finding the common elements of the solution set of a general system of variational inequalities and the common fixed point set of a countable family of nonexpansive mappings and zero points of an accretive operator in real smooth Banach spaces. Moreover, they [10] proposed an implicit composite extragradient-like method based on the Mann iteration method, the viscosity approximation method, and the Korpelevich extragradient method for solving a general system of variational inequalities with a hierarchical variational inequality constraint for countably many uniformly Lipschitzian pseudocontractive mappings and an accretive operator in a real Banach space. In [36, 38], Yao, Postolache, and Yao suggested a projected type algorithm and an extragradient algorithm for finding the common solutions of two variational inequalities and the common element of the set of fixed points of a pseudocontractive operator and the set of solutions of the variational inequality problem in Hilbert spaces, respectively. In [35, 37], Yao et al. introduced iterative algorithms for solving a split variational inequality and a fixed point problem that requires finding a solution of a generalized variational inequality whose image is a fixed point of a pseudocontractive operator or a fixed point of two quasi-pseudocontractive operators under a nonlinear transformation in Hilbert spaces. In [33, 34], Yao et al. constructed iterative algorithms for solving the split feasibility problem and the fixed point problem, the split equilibrium problems and fixed point problems involved in the pseudocontractive mappings in Hilbert spaces and proved their strong convergence.

Inspired and motivated by the above research work, we suggest an iterative approximation method for finding an element of the common solution set of HFPP (2), SEP (4)-(5), and SVI (7) involved in nonexpansive mappings. To our best knowledge, there is no further study on finding the element of the common solution set of HFPP (2), SEP (4)-(5), and SVI (7). When the mappings take different types of cases, we can obtain a corollary on the common element of the set of fixed points of a nonexpansive mapping, the solution set of a variational inequality and an equilibrium problem. So, our results presented here are new and very interesting.

The paper is organized as follows. In Sect. 2, we recall some concepts and lemmas which are needed in proving our main results. In Sect. 3, we suggest an iterative algorithm for solving the three different kinds of problems and prove its strong convergence. At last, the conclusion is given. 


\section{Preliminaries}

In this section, we list some fundamental results that are useful in the consequent analysis.

Let $H$ be a real Hilbert space, $C$ be a nonempty closed and convex subset of $H$.

Then, for all $x, y \in H$, the following inequalities hold:

$$
\|x-y\|^{2}=\|x\|^{2}-\|y\|^{2}-2\langle x-y, y\rangle, \quad\|x+y\|^{2} \leq\|x\|^{2}+2\langle y, x+y\rangle .
$$

A function $F: C \times C \rightarrow R$ is called an equilibrium function if it satisfies the following conditions:

(A1) $F(x, x)=0$ for all $x \in C$;

(A2) $F$ is monotone, i.e., $F(x, y)+F(y, x) \leq 0$ for all $x, y \in C$;

(A3) $\lim \sup _{t \downarrow 0} F(t z+(1-t) x, y) \leq F(x, y)$ for all $x, y, z \in C$;

(A4) for each $x \in C, y \mapsto F(x, y)$ is convex and lower semi-continuous;

(A5) Fix $r>0$ and $z \in C$, there exists a nonempty compact convex subset $K$ of $H$ and $x \in C \cap K$ such that

$$
F(y, x)+\frac{1}{r}\langle y-x, x-z\rangle<0, \quad \forall y \in C \backslash K
$$

Lemma 2.1 ([16]) Assume that $F: C \times C \rightarrow R$ is an equilibrium function. For $r>0$, define a mapping $R_{r, F}: H \rightarrow C$ as follows:

$$
R_{r, F}(x)=\left\{z \in C: F(x, y)+\frac{1}{r}\langle y-z, z-x\rangle \geq 0, \forall y \in C\right\}
$$

for all $x \in H$. Then the following hold:

(B1) $R_{r, F}$ is single-valued;

(B2) Fix $\left(R_{r, F}\right)=E P(F)$ and $E P(F)$ is a nonempty closed and convex subset of $C$;

(B3) $R_{r, F}$ is a firmly nonexpansive mapping, i.e.,

$$
\left\|R_{r, F}(x)-R_{r, F}(y)\right\|^{2} \leq\left\langle R_{r, F}(x)-R_{r, F}(y), x-y\right\rangle, \quad \forall x, y \in H . C .
$$

Lemma 2.2 Let $F: C \times C \rightarrow R$ be an equilibrium function, and let $R_{r, F}$ be defined as in Lemma 2.1 for $r>0$. Let $x, y \in H$ and $r_{1}, r_{2}>0$, then

$$
\left\|R_{r_{2}, F}(y)-R_{r_{1}, F}(x)\right\| \leq\|y-x\|+\left|\frac{r_{2}-r_{1}}{r_{2}}\right|\left\|R_{r_{2}, F}(y)-y\right\| .
$$

Lemma 2.3 ([32]) Let $\left\{a_{n}\right\}$ be a sequence of nonnegative real numbers such that

$$
a_{n+1} \leq\left(1-\alpha_{n}\right) a_{n}+\delta_{n}, \quad n \geq 0,
$$

where $\left\{\alpha_{n}\right\}$ is a sequence in $(0,1)$ and $\left\{\delta_{n}\right\}$ is a sequence in $R$ such that
(i) $\sum_{n=1}^{\infty} \alpha_{n}=\infty$;
(ii) $\quad \limsup _{n \rightarrow \infty} \frac{\delta_{n}}{\alpha_{n}} \leq 0$ or $\sum_{n=1}^{\infty}\left|\delta_{n}\right|<\infty$.

Then $\lim _{n \rightarrow \infty} a_{n}=0$. 
Lemma 2.4 Let $P_{C}$ denote the projection of $H$ onto $C$. It is known that $P_{C}$ is nonexpansive and the following inequalities hold:

$$
\begin{aligned}
& \left\|P_{C} x-P_{C} y\right\|^{2} \leq\left\langle x-y, P_{C} x-P_{C} y\right\rangle, \quad \forall x, y \in H, \\
& \|x-y\|^{2} \geq\left\|x-P_{C} x\right\|^{2}+\left\|y-P_{C} x\right\|^{2}, \quad \forall x \in H, y \in C, \\
& \left\|(x-y)-\left(P_{C} x-P_{C} y\right)\right\|^{2} \geq\|x-y\|^{2}-\left\|P_{C} x-P_{C} y\right\|^{2}, \quad \forall x, y \in H .
\end{aligned}
$$

Lemma 2.5 If $B$ is an $\alpha$-inverse-strongly monotone mapping of $C$ into $H$, and $\lambda \in[0,2 \alpha]$, then $I-\lambda B$ is a nonexpansive mapping.

Proof For any $w, u \in C_{1}$, we have

$$
\begin{aligned}
\|(I-\lambda B) w-(I-\lambda B) u\|^{2} & =\|(w-u)-\lambda(B w-B u)\|^{2} \\
& =\|w-u\|^{2}-2 \lambda\langle B w-B u, w-u\rangle+\lambda^{2}\|B w-B u\|^{2} \\
& \leq\|w-u\|^{2}+\lambda(\lambda-2 \alpha)\|B w-B u\|^{2} \\
& \leq\|w-u\|^{2},
\end{aligned}
$$

which implies that $I-\lambda B$ is nonexpansive, completing the proof.

Lemma 2.6 ([7]) Let $C$ be a nonempty closed convex subset of a real Hilbert space H. Let $B_{i}: C \rightarrow H$ be an $\alpha_{i}$-inverse-strongly monotone mapping, where $i \in\{1,2, \ldots, N\}$. Let $G$ : $C \rightarrow C$ be a mapping defined by

$$
\begin{aligned}
& G(x)=P_{C}\left(I-\lambda_{N} B_{N}\right) P_{C}\left(I-\lambda_{N-1} B_{N-1}\right) \cdots P_{C}\left(I-\lambda_{2} B_{2}\right) P_{C}\left(I-\lambda_{1} B_{1}\right) x, \quad \forall x \in C . \\
& \text { If } \lambda_{i} \in\left[0,2 \alpha_{i}\right], i=1,2, \ldots, N \text {, then } G: C \rightarrow C \text { is nonexpansive. }
\end{aligned}
$$

Proof Putting $T^{i}=P_{C}\left(I-\lambda_{i} B_{i}\right) P_{C}\left(I-\lambda_{i-1} B_{i-1}\right) \cdots P_{C}\left(I-\lambda_{2} B_{2}\right) P_{C}\left(I-\lambda_{1} B_{1}\right), i=1,2, \ldots, N$, and $T^{0}=I$, where $I$ is an identity mapping on $C$. Then $G=T^{N}$. For all $x, y \in C$, we have

$$
\begin{aligned}
\|G(x)-G(y)\| & =\left\|T^{N}(x)-T^{N}(y)\right\| \\
& =\left\|P_{C}\left(I-\lambda_{N} B_{N}\right) T^{N-1} x-P_{C}\left(I-\lambda_{N} B_{N}\right) T^{N-1} y\right\| \\
& \leq\left\|\left(I-\lambda_{N} B_{N}\right) T^{N-1} x-\left(I-\lambda_{N} B_{N}\right) T^{N-1} y\right\| \\
& \leq\left\|T^{N-1} x-T^{N-1} y\right\| \\
& \vdots \\
& \leq\|x-y\| .
\end{aligned}
$$

Then $G$ is nonexpansive, which completes the proof.

Lemma 2.7 ([8]) Let $U: C \rightarrow H$ be a $\tau$-Lipschitzian mapping, and let $F: C \rightarrow H$ be a $k$ Lipschitzian mapping and $\eta$-strongly monotone mapping, then, for $0 \leq \rho \tau<\mu \eta, \mu F-\rho U$ 
is $(\mu \eta-\rho \tau)$-strongly monotone, i.e.,

$$
\langle(\mu F-\rho U) x-(\mu F-\rho U) y, x-y\rangle \geq(\mu \eta-\rho \tau)\|x-y\|^{2}, \quad \forall x, y \in C .
$$

Lemma 2.8 ([26]) Suppose that $\lambda \in(0,1)$ and $\mu>0$. Let $F: C \rightarrow H$ be a -Lipschitzian and $\eta$-strongly monotone mapping. In association with a nonexpansive mapping $T: C \rightarrow C$, define the mapping $T^{\lambda}: C \rightarrow H$ by

$$
T^{\lambda}(x)=T(x)-\lambda \mu F T(x), \quad \forall x \in C .
$$

Then $T^{\lambda}$ is a contractive mapping with $\mu<\frac{2 \eta}{k^{2}}$, that is,

$$
\left\|T^{\lambda} x-T^{\lambda} y\right\| \leq(1-\lambda \nu)\|x-y\|, \quad \forall x, y \in C,
$$

where $v=1-\sqrt{1-\mu\left(2 \eta-\mu k^{2}\right)}$.

Lemma 2.9 ([24]) Each Hilbert space $H$ satisfies the Opial condition, that is, for any sequence $\left\{x_{n}\right\}$ with $x_{n}$ converging weakly to $x$, the inequality $\liminf _{n \rightarrow \infty}\left\|x_{n}-x\right\|<$ $\liminf _{n \rightarrow \infty}\left\|x_{n}-y\right\|$ holds for every $y \in H$ with $y \neq x$.

Lemma 2.10 ([4] Demiclosedness principle) Let $C$ be a closed convex subset of a real Hilbert space $H$, and let $T: C \rightarrow C$ be a nonexpansive mapping. Then $I-T$ is demiclosed at zero, that is, $x_{n}$ converges weakly to $x, x_{n}-T x_{n} \rightarrow 0$ implies $x=T x$.

\section{Main results}

Theorem 3.1 For $i \in\{1,2\}$, let $H_{i}$ be a real Hilbert space, $C_{i}$ be a nonempty closed convex subset of $H_{i}$, let $F_{i}: C_{i} \times C_{i} \rightarrow R$ be an equilibrium function. Let $A: H_{1} \rightarrow H_{2}$ be bounded linear operators with their adjoint operators $A^{*}$. Let $B_{i}$ be $\xi_{i}$-inverse-strongly monotone, respectively, where $i \in\{1,2, \ldots, N\}$. Let $F: C_{1} \rightarrow C_{1}$ be a k-Lipschitzian mapping and $\eta$ strongly monotone, and let $U: C_{1} \rightarrow C_{1}$ be a $\tau$-Lipschitzian mapping. Let $S, T: C_{1} \rightarrow C_{1}$ be two nonexpansive mappings such that $\Theta=\Gamma \cap \operatorname{Fix}(G) \cap \operatorname{Fix}(T) \neq \emptyset$. For a given $x_{0} \in C_{1}$ arbitrarily, let the iterative sequences $\left\{u_{n}\right\},\left\{y_{n}\right\}$, and $\left\{x_{n}\right\}$ be generated by

$$
\left\{\begin{array}{l}
u_{n}=R_{r_{n}, F_{1}}\left(x_{n}+\gamma A^{*}\left(R_{r_{n}, F_{2}}-I\right) A x_{n}\right), \\
y_{n}=P_{C_{1}}\left(I-\lambda_{N} B_{N}\right) P_{C_{1}}\left(I-\lambda_{N-1} B_{N-1}\right) \cdots P_{C_{1}}\left(I-\lambda_{2} B_{2}\right) P_{C_{1}}\left(I-\lambda_{1} B_{1}\right) u_{n}, \\
z_{n}=\beta_{n} S x_{n}+\left(1-\beta_{n}\right) y_{n}, \\
x_{n+1}=P_{C_{1}}\left[\alpha_{n} \rho U\left(x_{n}\right)+\left(I-\alpha_{n} \mu F\right)\left(T\left(z_{n}\right)\right)\right],
\end{array}\right.
$$

where $\left\{r_{n}\right\} \subset(0, \infty), \gamma \in\left(0,1 / L_{A}\right), L_{A}$ is the spectral radius of the operators $A^{*} A$. Suppose that the parameters satisfy $0<\mu<\frac{2 \eta}{k^{2}}, k \geq \eta, 0 \leq \rho \tau<\nu$, where $v=1-\sqrt{1-\mu(2 \eta-\mu k)^{2}}$, and $\left\{\alpha_{n}\right\},\left\{\beta_{n}\right\}$ are the sequences in $(0,1)$ satisfying the following conditions:

(i) $\lim _{n \rightarrow \infty} \alpha_{n}=0$ and $\sum_{n=0}^{\infty} \alpha_{n}=\infty, \sum_{n=1}^{\infty}\left|\alpha_{n-1}-\alpha_{n}\right|<\infty$;

(ii) $\lim \sup _{n \rightarrow \infty} \frac{\beta_{n}}{\alpha_{n}}=0, \beta_{n} \leq \alpha_{n}(n \geq 1)$ and $\sum_{n=1}^{\infty}\left|\beta_{n-1}-\beta_{n}\right|<\infty$;

(iii) $\liminf _{n \rightarrow \infty} r_{n}>0, \sum_{n=1}^{\infty}\left|r_{n-1}-r_{n}\right|<\infty$.

Then the sequence $\left\{x_{n}\right\}$ generated by (8) converges strongly to $w \in \Theta$. 
Proof Let $p \in \Theta$, i.e., $p \in \Gamma$, that is, $p=R_{r_{n}, F_{1}}(p)$ and $A p=R_{r_{n}, F_{2}}(A p)$. For convenience, we split the proof into several steps.

Step 1. We show that $\left\{x_{n}\right\},\left\{u_{n}\right\},\left\{y_{n}\right\},\left\{z_{n}\right\}$ are bounded.

First, by (8) and the expansiveness of $R_{r_{n}, F_{1}}$, we estimate

$$
\begin{aligned}
\left\|u_{n}-p\right\|^{2} & =\left\|R_{r_{n}, F_{1}}\left(x_{n}+\gamma A^{*}\left(R_{r_{n}, F_{2}}-I\right) A x_{n}\right)-p\right\|^{2} \\
& =\left\|R_{r_{n}, F_{1}}\left(x_{n}+\gamma A^{*}\left(R_{r_{n}, F_{2}}-I\right) A x_{n}\right)-R_{r_{n}, F_{1}}(p)\right\|^{2} \\
& \leq\left\|x_{n}+\gamma A^{*}\left(R_{r_{n}, F_{2}}-I\right) A x_{n}-p\right\|^{2} \\
& =\left\|x_{n}-p\right\|^{2}+\gamma^{2}\left\|A^{*}\left(R_{r_{n}, F_{2}}-I\right) A x_{n}\right\|^{2}+2 \gamma\left\langle x_{n}-p, A^{*}\left(R_{r_{n}, F_{2}}-I\right) A x_{n}\right\rangle .
\end{aligned}
$$

It follows from the definition of $L_{A}$ that

$$
\begin{aligned}
\gamma^{2} & \left\|A^{*}\left(R_{r_{n}, F_{2}}-I\right) A x_{n}\right\|^{2} \\
& =\gamma^{2}\left\langle\left(R_{r_{n}, F_{2}}-I\right) A x_{n}, A A^{*}\left(R_{r_{n}, F_{2}}-I\right) A x_{n}\right\rangle \\
& \leq L_{A} \gamma^{2}\left\|\left(R_{r_{n}, F_{2}}-I\right) A x_{n}\right\|^{2} .
\end{aligned}
$$

By using Lemma 2.4, we have

$$
\begin{aligned}
2 \gamma & \left\langle x_{n}-p, A^{*}\left(R_{r_{n}, F_{2}}-I\right) A x_{n}\right\rangle \\
& =2 \gamma\left\langle A\left(x_{n}-p\right),\left(R_{r_{n}, F_{2}}-I\right) A x_{n}\right\rangle \\
& =2 \gamma\left\langle A\left(x_{n}-p\right)+\left(R_{r_{n}, F_{2}}-I\right) A x_{n}-\left(R_{r_{n}, F_{2}}-I\right) A x_{n},\left(R_{r_{n}, F_{2}}-I\right) A x_{n}\right\rangle \\
& =2 \gamma\left\{\left\langle R_{r_{n}, F_{2}} A x_{n}-A p,\left(R_{r_{n}, F_{2}}-I\right) A x_{n}\right\rangle-\left\|\left(R_{r_{n}, F_{2}}-I\right) A x_{n}\right\|^{2}\right\} \\
& \leq 2 \gamma\left\{\frac{1}{2}\left\|\left(R_{r_{n}, F_{2}}-I\right) A x_{n}\right\|^{2}-\left\|\left(R_{r_{n}, F_{2}}-I\right) A x_{n}\right\|^{2}\right\} \\
& =-\gamma\left\|\left(R_{r_{n}, F_{2}}-I\right) A x_{n}\right\|^{2} .
\end{aligned}
$$

From (9)-(11) and $\gamma \in\left(0,1 / L_{A}\right)$ it follows that

$$
\left\|u_{n}-p\right\|^{2} \leq\left\|x_{n}-p\right\|^{2}+\gamma\left(L_{A} \gamma-1\right)\left\|\left(R_{r_{n}, F_{2}}-I\right) A x_{n}\right\|^{2} \leq\left\|x_{n}-p\right\|^{2} .
$$

It follows from (8), (12), and Lemma 2.6 that we have

$$
\left\|y_{n}-p\right\|=\left\|T^{N} u_{n}-T^{N} p\right\| \leq\left\|u_{n}-p\right\| \leq\left\|x_{n}-p\right\|
$$

Next, we prove that the sequence $\left\{x_{n}\right\}$ is bounded. Note $\beta_{n} \leq \alpha_{n}$ for all $n \geq 1$. Put $V_{n}=$ $\alpha_{n} \rho U\left(x_{n}\right)+\left(I-\alpha_{n} \mu F\right)\left(T\left(z_{n}\right)\right)$,

from (8), we get

$$
\begin{aligned}
\left\|x_{n+1}-p\right\| & =\left\|P_{C_{1}}\left[\alpha_{n} \rho U\left(x_{n}\right)+\left(I-\alpha_{n} \mu F\right)\left(T\left(z_{n}\right)\right)\right]-p\right\| \\
& \leq \alpha_{n}\left\|\rho U\left(x_{n}\right)-\mu F(p)\right\|+\left\|\left(I-\alpha_{n} \mu F\right)\left(T\left(z_{n}\right)\right)-\left(I-\alpha_{n} \mu F\right)(T(p))\right\| \\
& =\alpha_{n}\left\|\rho U\left(x_{n}\right)-\rho U(p)+(\rho U-\mu F)(p)\right\|
\end{aligned}
$$




$$
\begin{aligned}
& +\left\|\left(I-\alpha_{n} \mu F\right)\left(T\left(z_{n}\right)\right)-\left(I-\alpha_{n} \mu F\right)(T(p))\right\| \\
\leq & \alpha_{n} \rho \tau\left\|x_{n}-p\right\|+\alpha_{n}\|(\rho U-\mu F)(p)\|+\left(1-\alpha_{n} v\right)\left\|z_{n}-p\right\| \\
\leq & \alpha_{n} \rho \tau\left\|x_{n}-p\right\|+\alpha_{n}\|(\rho U-\mu F)(p)\| \\
& +\left(1-\alpha_{n} v\right)\left\|\beta_{n} S x_{n}+\left(1-\beta_{n}\right) y_{n}-p\right\| \\
\leq & \alpha_{n} \rho \tau\left\|x_{n}-p\right\|+\alpha_{n}\|(\rho U-\mu F)(p)\| \\
& +\left(1-\alpha_{n} v\right)\left(\beta_{n}\left\|S x_{n}-S p\right\|+\beta_{n}\|S p-p\|+\left(1-\beta_{n}\right)\left\|y_{n}-p\right\|\right) \\
\leq & \alpha_{n} \rho \tau\left\|x_{n}-p\right\|+\alpha_{n}\|(\rho U-\mu F)(p)\| \\
& +\left(1-\alpha_{n} v\right)\left(\beta_{n}\left\|x_{n}-p\right\|+\beta_{n}\|S p-p\|+\left(1-\beta_{n}\right)\left\|x_{n}-p\right\|\right) \\
\leq & \left(1-\alpha_{n}(v-\rho \tau)\right)\left\|x_{n}-p\right\|+\alpha_{n}\|(\rho U-\mu F)(p)\| \\
& +\left(1-\alpha_{n} v\right) \beta_{n}\|S p-p\| \\
\leq & \left(1-\alpha_{n}(v-\rho \tau)\right)\left\|x_{n}-p\right\|+\alpha_{n}\|(\rho U-\mu F)(p)\|+\beta_{n}\|S p-p\| \\
\leq & \left(1-\alpha_{n}(v-\rho \tau)\right)\left\|x_{n}-p\right\|+\alpha_{n}(\|(\rho U-\mu F)(p)\|+\|S p-p\|) \\
\leq & \left(1-\alpha_{n}(v-\rho \tau)\right)\left\|x_{n}-p\right\|+\frac{\alpha_{n}(v-\rho \tau)}{v-\rho \tau}(\|(\rho U-\mu F)(p)\|+\|S p-p\|) \\
\leq & \max \left\{\left\|x_{0}-p\right\|, \frac{1}{v-\rho \tau}(\|(\rho U-\mu F)(p)\|+\|S p-p\|)\right\} .
\end{aligned}
$$

So $\left\{x_{n}\right\}$ is bounded, and consequently we can deduce that $\left\{u_{n}\right\},\left\{y_{n}\right\},\left\{z_{n}\right\}$ are also bounded.

Step 2. We will show the following:
(a) $\lim _{n \rightarrow \infty}\left\|x_{n+1}-x_{n}\right\|=0$;
(b) $\lim _{n \rightarrow \infty}\left\|u_{n}-x_{n}\right\|=0$;
(c) $\lim _{n \rightarrow \infty}\left\|u_{n}-y_{n}\right\|=0$.

Noting $u_{n}=R_{r_{n}, F_{1}}\left(x_{n}+\gamma A^{*}\left(R_{r_{n}, F_{2}}-I\right) A x_{n}\right)$ and $u_{n-1}=R_{r_{n-1}, F_{1}}\left(x_{n-1}+\gamma A^{*}\left(R_{r_{n-1}, F_{2}}-\right.\right.$ I) $A x_{n-1}$ ), from Lemma 2.2, we have

$$
\begin{aligned}
\| u_{n}- & u_{n-1} \| \\
= & \left\|R_{r_{n}, F_{1}} v_{n}-R_{r_{n-1}, F_{1}} v_{n-1}\right\| \\
\leq & \left.\| x_{n}-x_{n-1}+\gamma A^{*}\left[\left(R_{r_{n}, F_{2}}-I\right) A x_{n}-\left(R_{r_{n-1}, F_{2}}-I\right) A x_{n-1}\right)\right] \| \\
& +\left|1-\frac{r_{n-1}}{r_{n}}\right|\left\|R_{r_{n}, F_{1}}\left(x_{n}+\gamma A^{*}\left(R_{r_{n}, F_{2}}-I\right) A x_{n}\right)-x_{n}-\gamma A^{*}\left(R_{r_{n}, F_{2}}-I\right) A x_{n}\right\| \\
\leq & \left\|x_{n}-x_{n-1}-\gamma A^{*} A\left(x_{n}-x_{n-1}\right)\right\|+\gamma\left\|A^{*}\right\|\left\|R_{r_{n}, F_{2}} A x_{n}-R_{r_{n-1}, F_{2}} A x_{n-1}\right\| \\
& +\left|1-\frac{r_{n-1}}{r_{n}}\right| \delta_{n-1} \\
\leq & \left\{\left\|x_{n}-x_{n-1}\right\|^{2}-2 \gamma\left\|A x_{n}-A x_{n-1}\right\|^{2}+\gamma^{2}\|A\|^{4}\left\|x_{n}-x_{n-1}\right\|^{2}\right\}^{\frac{1}{2}} \\
& +\gamma\|A\|\left\{\left\|A x_{n}-A x_{n-1}\right\|+\left|1-\frac{r_{n-1}}{r_{n}}\right|\left\|R_{r_{n}, F_{2}} A x_{n}-A x_{n}\right\|\right\}+\left|1-\frac{r_{n-1}}{r_{n}}\right| \delta_{n-1} \\
\leq & \left(1-2 \gamma\|A\|^{2}+\gamma^{2}\|A\|^{4}\right)^{\frac{1}{2}}\left\|x_{n}-x_{n-1}\right\|+\gamma\|A\|^{2}\left\|x_{n}-x_{n-1}\right\|
\end{aligned}
$$




$$
\begin{aligned}
& +\left|1-\frac{r_{n-1}}{r_{n}}\right| \gamma\|A\| \sigma_{n-1}+\left|1-\frac{r_{n-1}}{r_{n}}\right| \delta_{n-1} \\
= & \left\|x_{n}-x_{n-1}\right\|+\left|1-\frac{r_{n-1}}{r_{n}}\right|\left(\gamma\|A\| \sigma_{n-1}+\delta_{n-1}\right),
\end{aligned}
$$

where

$$
\begin{aligned}
& \delta_{n-1}=\left\|R_{r_{n}, F_{1}}\left(x_{n}+\gamma A^{*}\left(R_{r_{n}, F_{2}}-I\right) A x_{n}\right)-\left(x_{n}+\gamma A^{*}\left(R_{r_{n}, F_{2}}-I\right) A x_{n}\right)\right\|, \\
& \sigma_{n-1}=\left\|R_{r_{n}, F_{2}} A x_{n}-A x_{n}\right\| .
\end{aligned}
$$

So, from Lemma 2.6, we have

$$
\begin{aligned}
\left\|y_{n}-y_{n-1}\right\| & =\left\|G\left(u_{n}\right)-G\left(u_{n-1}\right)\right\| \leq\left\|u_{n}-u_{n-1}\right\| \\
& \leq\left\|x_{n}-x_{n-1}\right\|+\left|1-\frac{r_{n-1}}{r_{n}}\right|\left(\gamma\|A\| \sigma_{n-1}+\delta_{n-1}\right) .
\end{aligned}
$$

Then from (16) we get

$$
\begin{aligned}
\left\|z_{n}-z_{n-1}\right\|= & \left\|\beta_{n} S x_{n}+\left(1-\beta_{n}\right) y_{n}-\beta_{n-1} S x_{n-1}-\left(1-\beta_{n-1}\right) y_{n-1}\right\| \\
\leq & \beta_{n}\left\|x_{n}-x_{n-1}\right\|+\left|\beta_{n}-\beta_{n-1}\right|\left(\left\|S x_{n-1}\right\|+\left\|y_{n-1}\right\|\right)+\left(1-\beta_{n}\right)\left\|y_{n}-y_{n-1}\right\| \\
\leq & \beta_{n}\left\|x_{n}-x_{n-1}\right\|+\left|\beta_{n}-\beta_{n-1}\right|\left(\left\|S x_{n-1}\right\|+\left\|y_{n-1}\right\|\right) \\
& +\left(1-\beta_{n}\right)\left\{\left\|x_{n}-x_{n-1}\right\|+\left|1-\frac{r_{n-1}}{r_{n}}\right|\left(\gamma\|A\| \sigma_{n-1}+\delta_{n-1}\right)\right\} \\
\leq & \left\|x_{n}-x_{n-1}\right\|+\left|1-\frac{r_{n-1}}{r_{n}}\right|\left(\gamma\|A\| \sigma_{n-1}+\delta_{n-1}\right) \\
& +\left|\beta_{n}-\beta_{n-1}\right|\left(\left\|S x_{n-1}\right\|+\left\|y_{n-1}\right\|\right) .
\end{aligned}
$$

Next, by Lemma 2.8, we estimate

$$
\begin{aligned}
&\left\|x_{n+1}-x_{n}\right\| \\
&=\left\|P_{C}\left[V_{n}\right]-P_{C}\left[V_{n-1}\right]\right\| \\
& \leq \| \alpha_{n} \rho\left(U\left(x_{n}\right)-U\left(x_{n-1}\right)\right)+\left(\alpha_{n}-\alpha_{n-1}\right) \rho U\left(x_{n-1}\right)+\left(I-\alpha_{n} \mu F\right)\left(T\left(z_{n}\right)\right) \\
& \quad-\left(I-\alpha_{n} \mu F\right)\left(T\left(z_{n-1}\right)\right)+\left(I-\alpha_{n} \mu F\right)\left(T\left(z_{n-1}\right)\right)-\left(I-\alpha_{n-1} \mu F\right)\left(T\left(z_{n-1}\right)\right) \| \\
& \leq \alpha_{n} \rho \tau\left\|x_{n}-x_{n-1}\right\|+\left|\alpha_{n}-\alpha_{n-1}\right|\left(\left\|\rho U\left(x_{n-1}\right)\right\|+\left\|\mu F\left(T\left(z_{n-1}\right)\right)\right\|\right) \\
&+\left(1-\alpha_{n} \nu\right)\left\|z_{n}-z_{n-1}\right\| .
\end{aligned}
$$

From (17) and (18), we get

$$
\begin{aligned}
& \left\|x_{n+1}-x_{n}\right\| \\
& \leq \alpha_{n} \rho \tau\left\|x_{n}-x_{n-1}\right\|+\left|\alpha_{n}-\alpha_{n-1}\right|\left(\left\|\rho U\left(x_{n-1}\right)\right\|+\left\|\mu F\left(T\left(z_{n-1}\right)\right)\right\|\right) \\
& \quad+\left(1-\alpha_{n} \nu\right)\left\{\left\|x_{n}-x_{n-1}\right\|+\left|1-\frac{r_{n-1}}{r_{n}}\right|\left(\gamma\|A\| \sigma_{n-1}+\delta_{n-1}\right)\right.
\end{aligned}
$$




$$
\begin{aligned}
& \left.+\left|\beta_{n}-\beta_{n-1}\right|\left(\left\|S x_{n-1}\right\|+\left\|z_{n-1}\right\|\right)\right\} \\
\leq & \left(1-(\nu-\rho \tau) \alpha_{n}\right)\left\|x_{n}-x_{n-1}\right\|+\left|\alpha_{n}-\alpha_{n-1}\right|\left(\left\|\rho U\left(x_{n-1}\right)\right\|+\left\|\mu F\left(T\left(z_{n-1}\right)\right)\right\|\right) \\
& +\left|1-\frac{r_{n-1}}{r_{n}}\right|\left(\gamma\|A\| \sigma_{n-1}+\delta_{n-1}\right)+\left|\beta_{n}-\beta_{n-1}\right|\left(\left\|S x_{n-1}\right\|+\left\|z_{n-1}\right\|\right) \\
\leq & \left(1-(v-\rho \tau) \alpha_{n}\right)\left\|x_{n}-x_{n-1}\right\|+M\left(\left|\alpha_{n}-\alpha_{n-1}\right|+\frac{1}{\varepsilon}\left|r_{n-1}-r_{n}\right|+\left|\beta_{n}-\beta_{n-1}\right|\right),
\end{aligned}
$$

where $\quad M=\max \left\{\sup _{n \geq 1}\left(\left\|\rho U\left(x_{n-1}\right)\right\|+\left\|\mu F\left(T\left(z_{n-1}\right)\right)\right\|\right), \sup _{n \geq 1}\left(\gamma\|A\| \sigma_{n-1}+\delta_{n-1}\right)\right.$, $\left.\sup _{n \geq 1}\left(\left\|S x_{n-1}\right\|+\left\|z_{n-1}\right\|\right)\right\}$. And $\varepsilon$ is a real number such that $0<\varepsilon<r_{n}$. So, it follows from Conditions (i)-(iii) and Lemma 2.3 that

$$
\lim _{n \rightarrow \infty}\left\|x_{n+1}-x_{n}\right\|=0
$$

Next, we show that $\lim _{n \rightarrow \infty}\left\|u_{n}-x_{n}\right\|=0$. In view of (8), (9), (12), and (13), we obtain

$$
\begin{aligned}
& \left\|x_{n+1}-p\right\|^{2}=\left\langle P_{C}\left[V_{n}\right]-p, x_{n+1}-p\right\rangle \\
& =\left\langle P_{C}\left[V_{n}\right]-V_{n}, P_{C}\left[V_{n}\right]-p\right\rangle+\left\langle V_{n}-p, x_{n+1}-p\right\rangle \\
& \leq\left\langle\alpha_{n}\left(\rho U\left(x_{n}\right)-\mu F(p)\right)+\left(I-\alpha_{n} \mu F\right)\left(T\left(z_{n}\right)\right)\right. \\
& \left.-\left(I-\alpha_{n} \mu F\right)(T(p)), x_{n+1}-p\right) \\
& =\left\langle\alpha_{n} \rho\left(U\left(x_{n}\right)-U(p)\right), x_{n+1}-p\right\rangle+\alpha_{n}\left\langle\rho U(p)-\mu F(p), x_{n+1}-p\right\rangle \\
& +\left\langle\left(I-\alpha_{n} \mu F\right)\left(T\left(z_{n}\right)\right)-\left(I-\alpha_{n} \mu F\right)(T(p)), x_{n+1}-p\right\rangle \\
& \leq \alpha_{n} \rho \tau\left\|x_{n}-p\right\|\left\|x_{n+1}-p\right\|+\alpha_{n}\left\langle\rho U(p)-\mu F(p), x_{n+1}-p\right\rangle \\
& +\left(1-\alpha_{n} v\right)\left\|z_{n}-p\right\|\left\|x_{n+1}-p\right\| \\
& \leq \frac{\alpha_{n} \rho \tau}{2}\left(\left\|x_{n}-p\right\|^{2}-\left\|x_{n+1}-p\right\|^{2}\right)+\alpha_{n}\left\langle\rho U(p)-\mu F(p), x_{n+1}-p\right\rangle \\
& +\frac{\left(1-\alpha_{n} v\right)}{2}\left(\left\|z_{n}-p\right\|^{2}-\left\|x_{n+1}-p\right\|^{2}\right) \\
& \leq \frac{\left(1-\alpha_{n}(\nu-\rho \tau)\right)}{2}\left\|x_{n+1}-p\right\|^{2}+\alpha_{n}\left\langle\rho U(p)-\mu F(p), x_{n+1}-p\right\rangle \\
& +\frac{\alpha_{n} \rho \tau}{2}\left\|x_{n}-p\right\|^{2}+\frac{\left(1-\alpha_{n} \nu\right)}{2}\left\|z_{n}-p\right\|^{2} \\
& \leq \frac{\left(1-\alpha_{n}(\nu-\rho \tau)\right)}{2}\left\|x_{n+1}-p\right\|^{2}+\alpha_{n}\left\langle\rho U(p)-\mu F(p), x_{n+1}-p\right\rangle \\
& +\frac{\alpha_{n} \rho \tau}{2}\left\|x_{n}-p\right\|^{2}+\frac{\left(1-\alpha_{n} \nu\right)}{2}\left(\beta_{n}\left\|S x_{n}-p\right\|^{2}+\left(1-\beta_{n}\right)\left\|y_{n}-p\right\|^{2}\right) \text {. }
\end{aligned}
$$

From the above inequality and (12), (13), we get

$$
\begin{aligned}
\left\|x_{n+1}-p\right\|^{2} \leq & \frac{\alpha_{n} \rho \tau}{1+\alpha_{n}(v-\rho \tau)}\left\|x_{n}-p\right\|^{2}+\frac{2 \alpha_{n}}{1+\alpha_{n}(v-\rho \tau)}\left\langle\rho U(p)-\mu F(p), x_{n+1}-p\right\rangle \\
& +\frac{\left(1-\alpha_{n} \nu\right) \beta_{n}}{1+\alpha_{n}(v-\rho \tau)}\left\|S x_{n}-p\right\|^{2} \\
& +\frac{\left(1-\alpha_{n} \nu\right)\left(1-\beta_{n}\right)}{1+\alpha_{n}(v-\rho \tau)}\left\{\left\|x_{n}-p\right\|^{2}+\gamma\left(L_{A} \gamma-1\right)\left\|\left(R_{r_{n}, F_{2}}-I\right) A x_{n}\right\|^{2}\right\}
\end{aligned}
$$




$$
\begin{aligned}
\leq & \frac{\alpha_{n} \rho \tau}{1+\alpha_{n}(\nu-\rho \tau)}\left\|x_{n}-p\right\|^{2}+\frac{2 \alpha_{n}}{1+\alpha_{n}(v-\rho \tau)}\left\langle\rho U(p)-\mu F(p), x_{n+1}-p\right\rangle \\
& +\frac{\left(1-\alpha_{n} \nu\right) \beta_{n}}{1+\alpha_{n}(v-\rho \tau)}\left\|S x_{n}-p\right\|^{2}+\left\|x_{n}-p\right\|^{2} \\
& +\frac{\left(1-\alpha_{n} v\right)\left(1-\beta_{n}\right)}{1+\alpha_{n}(v-\rho \tau)}\left\{\gamma\left(L_{A} \gamma-1\right)\left\|\left(R_{r_{n}, F_{2}}-I\right) A x_{n}\right\|^{2}\right\},
\end{aligned}
$$

which means that

$$
\begin{aligned}
& \frac{\left(1-\alpha_{n} \nu\right)\left(1-\beta_{n}\right)}{1+\alpha_{n}(v-\rho \tau)}\left\{\gamma\left(1-L_{A} \gamma\right)\left\|\left(R_{r_{n}, F_{2}}-I\right) A x_{n}\right\|^{2}\right\} \\
& \leq \frac{\alpha_{n} \rho \tau}{1+\alpha_{n}(v-\rho \tau)}\left\|x_{n}-p\right\|^{2}+\frac{2 \alpha_{n}}{1+\alpha_{n}(v-\rho \tau)}\left\{\rho U(p)-\mu F(p), x_{n+1}-p\right\rangle \\
& \quad+\frac{\left(1-\alpha_{n} v\right) \beta_{n}}{1+\alpha_{n}(v-\rho \tau)}\left\|S x_{n}-p\right\|^{2}+\left\|x_{n}-p\right\|^{2}-\left\|x_{n+1}-p\right\|^{2} \\
& \leq \frac{\alpha_{n} \rho \tau}{1+\alpha_{n}(v-\rho \tau)}\left\|x_{n}-p\right\|^{2}+\frac{2 \alpha_{n}}{1+\alpha_{n}(v-\rho \tau)}\left\langle\rho U(p)-\mu F(p), x_{n+1}-p\right\rangle \\
&+\beta_{n}\left\|S x_{n}-p\right\|^{2}+\left(\left\|x_{n}-p\right\|+\left\|x_{n+1}-p\right\|\right)\left\|x_{n+1}-x_{n}\right\| .
\end{aligned}
$$

Since $\alpha_{n} \rightarrow 0, \beta_{n} \rightarrow 0$ and $\lim _{n \rightarrow \infty}\left\|x_{n+1}-x_{n}\right\|=0$, we obtain

$$
\lim _{n \rightarrow \infty}\left\|\left(R_{r_{n}, F_{2}}-I\right) A x_{n}\right\|=0 .
$$

And since $R_{r_{n}, F_{1}}$ is firmly nonexpansive, from (8) we get

$$
\begin{aligned}
\| u_{n}- & p \|^{2} \\
= & \left\|R_{r_{n}, F_{1}}\left(x_{n}+\gamma A^{*}\left(R_{r_{n}, F_{2}}-I\right) A x_{n}\right)-p\right\|^{2} \\
= & \left\|R_{r_{n}, F_{1}}\left(x_{n}+\gamma A^{*}\left(R_{r_{n}, F_{2}}-I\right) A x_{n}\right)-R_{r_{n}, F_{1}}(p)\right\|^{2} \\
\leq & \left\langle u_{n}-p, x_{n}+\gamma A^{*}\left(R_{r_{n}, F_{2}}-I\right) A x_{n}-p\right\rangle \\
= & \frac{1}{2}\left\{\left\|u_{n}-p\right\|^{2}+\left\|x_{n}+\gamma A^{*}\left(R_{r_{n}, F_{2}}-I\right) A x_{n}-p\right\|^{2}\right. \\
& \left.-\left\|u_{n}-p-\left[x_{n}+\gamma A^{*}\left(R_{r_{n}, F_{2}}-I\right) A x_{n}-p\right]\right\|^{2}\right\} \\
= & \frac{1}{2}\left\{\left\|u_{n}-p\right\|^{2}+\left\|x_{n}+\gamma A^{*}\left(R_{r_{n}, F_{2}}-I\right) A x_{n}-p\right\|^{2}\right. \\
& \left.-\left\|u_{n}-x_{n}-\gamma A^{*}\left(R_{r_{n}, F_{2}}-I\right) A x_{n}\right\|^{2}\right\} \\
= & \frac{1}{2}\left\{\left\|u_{n}-p\right\|^{2}+\left\|x_{n}-p\right\|^{2}+2 \gamma\left\langle x_{n}-p, A^{*}\left(R_{r_{n}, F_{2}}-I\right) A x_{n}\right\rangle\right. \\
& +\gamma^{2}\left\|A^{*}\left(R_{r_{n}, F_{2}}-I\right) A x_{n}\right\|^{2} \\
& \left.-\left[\left\|u_{n}-x_{n}\right\|^{2}-2 \gamma\left\langle u_{n}-x_{n}, A^{*}\left(R_{r_{n}, F_{2}}-I\right) A x_{n}\right\rangle+\gamma^{2}\left\|A^{*}\left(R_{r_{n}, F_{2}}-I\right) A x_{n}\right\|^{2}\right]\right\} \\
= & \frac{1}{2}\left\{\left\|u_{n}-p\right\|^{2}+\left\|x_{n}-p\right\|^{2}+2 \gamma\left\langle u_{n}-p, A^{*}\left(R_{r_{n}, F_{2}}-I\right) A x_{n}\right\rangle-\left\|u_{n}-x_{n}\right\|^{2}\right\},
\end{aligned}
$$

which implies that

$$
\left\|u_{n}-p\right\|^{2} \leq\left\|x_{n}-p\right\|^{2}-\left\|u_{n}-x_{n}\right\|^{2}+2 \gamma\left\|A\left(u_{n}-p\right)\right\|\left\|\left(R_{r_{n}, F_{2}}-I\right) A x_{n}\right\| .
$$


So, from (21) and (25) we have

$$
\begin{aligned}
\left\|x_{n+1}-p\right\|^{2} & \\
\leq & \frac{\left(1-\alpha_{n}(\nu-\rho \tau)\right)}{2}\left\|x_{n+1}-p\right\|^{2}+\alpha_{n}\left\langle\rho U(p)-\mu F(p), x_{n+1}-p\right\rangle \\
& +\frac{\alpha_{n} \rho \tau}{2}\left\|x_{n}-p\right\|^{2}+\frac{\left(1-\alpha_{n} \nu\right)}{2}\left(\beta_{n}\left\|S x_{n}-p\right\|^{2}+\left(1-\beta_{n}\right)\left\|u_{n}-p\right\|^{2}\right) \\
\leq & \frac{\left(1-\alpha_{n}(\nu-\rho \tau)\right)}{2}\left\|x_{n+1}-p\right\|^{2}+\alpha_{n}\left\langle\rho U(p)-\mu F(p), x_{n+1}-p\right\rangle+\frac{\alpha_{n} \rho \tau}{2}\left\|x_{n}-p\right\|^{2} \\
& +\frac{\left(1-\alpha_{n} \nu\right)}{2}\left\{\beta_{n}\left\|S x_{n}-p\right\|^{2}+\left(1-\beta_{n}\right)\left(\left\|x_{n}-p\right\|^{2}-\left\|u_{n}-x_{n}\right\|^{2}\right.\right. \\
& \left.\left.+2 \gamma\left\|A\left(u_{n}-p\right)\right\|\left\|\left(R_{r_{n}, F_{2}}-I\right) A x_{n}\right\|\right)\right\},
\end{aligned}
$$

which implies that

$$
\begin{aligned}
\left\|x_{n+1}-p\right\|^{2} & \\
\leq & \frac{\alpha_{n} \rho \tau}{1+\alpha_{n}(v-\rho \tau)}\left\|x_{n}-p\right\|^{2}+\frac{2 \alpha_{n}}{1+\alpha_{n}(v-\rho \tau)}\left\langle\rho U(p)-\mu F(p), x_{n+1}-p\right\rangle \\
& +\frac{\left(1-\alpha_{n} \nu\right) \beta_{n}}{1+\alpha_{n}(v-\rho \tau)}\left\|S x_{n}-p\right\|^{2}+\frac{\left(1-\alpha_{n} v\right)\left(1-\beta_{n}\right)}{1+\alpha_{n}(v-\rho \tau)}\left\{\left\|x_{n}-p\right\|^{2}-\left\|u_{n}-x_{n}\right\|^{2}\right. \\
& \left.+2 \gamma\left\|A\left(u_{n}-p\right)\right\|\left\|\left(R_{r_{n}, F_{2}}-I\right) A x_{n}\right\|\right\} \\
\leq & \frac{\alpha_{n} \rho \tau}{1+\alpha_{n}(v-\rho \tau)}\left\|x_{n}-p\right\|^{2}+\frac{2 \alpha_{n}}{1+\alpha_{n}(v-\rho \tau)}\left\langle\rho U(p)-\mu F(p), x_{n+1}-p\right\rangle \\
& +\frac{\left(1-\alpha_{n} v\right) \beta_{n}}{1+\alpha_{n}(v-\rho \tau)}\left\|S x_{n}-p\right\|^{2}+\left\|x_{n}-p\right\|^{2} \\
& +\frac{\left(1-\alpha_{n} \nu\right)\left(1-\beta_{n}\right)}{1+\alpha_{n}(v-\rho \tau)}\left\{-\left\|u_{n}-x_{n}\right\|^{2}+2 \gamma\left\|A\left(u_{n}-p\right)\right\|\left\|\left(R_{r_{n}, F_{2}}-I\right) A x_{n}\right\|\right\} .
\end{aligned}
$$

Hence

$$
\begin{aligned}
& \frac{\left(1-\alpha_{n} v\right)\left(1-\beta_{n}\right)}{1+\alpha_{n}(v-\rho \tau)}\left\|u_{n}-x_{n}\right\|^{2} \\
& \leq \frac{\alpha_{n} \rho \tau}{1+\alpha_{n}(\nu-\rho \tau)}\left\|x_{n}-p\right\|^{2}+\frac{2 \alpha_{n}}{1+\alpha_{n}(v-\rho \tau)}\left\langle\rho U(p)-\mu F(p), x_{n+1}-p\right\rangle \\
& +\frac{\left(1-\alpha_{n} \nu\right) \beta_{n}}{1+\alpha_{n}(\nu-\rho \tau)}\left\|S x_{n}-p\right\|^{2}+\left\|x_{n}-p\right\|^{2}-\left\|x_{n+1}-p\right\|^{2} \\
& +\frac{2\left(1-\alpha_{n} \nu\right)\left(1-\beta_{n}\right) \gamma}{1+\alpha_{n}(v-\rho \tau)}\left\|A\left(u_{n}-p\right)\right\|\left\|\left(R_{r_{n}, F_{2}}-I\right) A x_{n}\right\| \\
& \leq \frac{\alpha_{n} \rho \tau}{1+\alpha_{n}(\nu-\rho \tau)}\left\|x_{n}-p\right\|^{2}+\frac{2 \alpha_{n}}{1+\alpha_{n}(\nu-\rho \tau)}\left\langle\rho U(p)-\mu F(p), x_{n+1}-p\right\rangle \\
& +\frac{\left(1-\alpha_{n} \nu\right) \beta_{n}}{1+\alpha_{n}(\nu-\rho \tau)}\left\|S x_{n}-p\right\|^{2}+\left(\left\|x_{n}-p\right\|+\left\|x_{n+1}-p\right\|\right)\left\|x_{n+1}-x_{n}\right\| .
\end{aligned}
$$


Since $\lim _{n \rightarrow \infty} \alpha_{n}=0, \lim _{n \rightarrow \infty} \beta_{n}=0, \lim _{n \rightarrow \infty}\left\|x_{n+1}-x_{n}\right\|=0$, and $\lim _{n \rightarrow \infty} \|\left(R_{r_{n}, F_{2}}-\right.$ I) $A x_{n} \|=0$, we have

$$
\lim _{n \rightarrow \infty}\left\|u_{n}-x_{n}\right\|=0
$$

Then, by Lemma 2.5 and Lemma 2.6, we obtain

$$
\begin{aligned}
& \left\|T^{N} u_{n}-T^{N} p\right\|^{2} \\
& \quad=\left\|P_{C_{1}}\left(I-\lambda_{N} B_{N}\right) T^{N-1} u_{n}-P_{C_{1}}\left(I-\lambda_{N} B_{N}\right) T^{N-1} p\right\|^{2} \\
& \quad \leq\left\|\left(I-\lambda_{N} B_{N}\right) T^{N-1} u_{n}-\left(I-\lambda_{N} B_{N}\right) T^{N-1} p\right\|^{2} \\
& \quad \leq\left\|T^{N-1} u_{n}-T^{N-1} p\right\|^{2}+\lambda_{N}\left(\lambda_{N}-2 \xi_{N}\right)\left\|B_{N} T^{N-1} u_{n}-B_{N} T^{N-1} p\right\|^{2} \\
& \quad \leq\left\|u_{n}-p\right\|^{2}+\sum_{i=1}^{N} \lambda_{i}\left(\lambda_{i}-2 \xi_{i}\right)\left\|B_{i} T^{i-1} u_{n}-B_{i} T^{i-1} p\right\|^{2} \\
& \quad \leq\left\|x_{n}-p\right\|^{2}+\sum_{i=1}^{N} \lambda_{i}\left(\lambda_{i}-2 \xi_{i}\right)\left\|B_{i} T^{i-1} u_{n}-B_{i} T^{i-1} p\right\|^{2} .
\end{aligned}
$$

From (21), we obtain

$$
\begin{aligned}
\left\|x_{n+1}-p\right\|^{2} & \\
\leq & \frac{\alpha_{n} \rho \tau}{1+\alpha_{n}(v-\rho \tau)}\left\|x_{n}-p\right\|^{2}+\frac{2 \alpha_{n}}{1+\alpha_{n}(v-\rho \tau)}\left\langle\rho U(p)-\mu F(p), x_{n+1}-p\right\rangle \\
& +\frac{\left(1-\alpha_{n} v\right) \beta_{n}}{1+\alpha_{n}(v-\rho \tau)}\left\|S x_{n}-p\right\|^{2} \\
& +\frac{\left(1-\alpha_{n} v\right)\left(1-\beta_{n}\right)}{1+\alpha_{n}(v-\rho \tau)}\left\{\left\|x_{n}-p\right\|^{2}+\sum_{i=1}^{N} \lambda_{i}\left(\lambda_{i}-2 \xi_{i}\right)\left\|B_{i} T^{i-1} u_{n}-B_{i} T^{i-1} p\right\|^{2}\right\} \\
\leq & \frac{\alpha_{n} \rho \tau}{1+\alpha_{n}(v-\rho \tau)}\left\|x_{n}-p\right\|^{2}+\frac{2 \alpha_{n}}{1+\alpha_{n}(v-\rho \tau)}\left\langle\rho U(p)-\mu F(p), x_{n+1}-p\right\rangle \\
& +\frac{\left(1-\alpha_{n} \nu\right) \beta_{n}}{1+\alpha_{n}(v-\rho \tau)}\left\|S x_{n}-p\right\|^{2} \\
& +\left\|x_{n}-p\right\|^{2}+\frac{\left(1-\alpha_{n} v\right)\left(1-\beta_{n}\right)}{1+\alpha_{n}(v-\rho \tau)}\left\{\sum_{i=1}^{N} \lambda_{i}\left(\lambda_{i}-2 \xi_{i}\right)\left\|B_{i} T^{i-1} u_{n}-B_{i} T^{i-1} p\right\|^{2}\right\},
\end{aligned}
$$

which implies that

$$
\begin{aligned}
& \frac{\left(1-\alpha_{n} \nu\right)\left(1-\beta_{n}\right)}{1+\alpha_{n}(v-\rho \tau)}\left\{\sum_{i=1}^{N} \lambda_{i}\left(2 \xi_{i}-\lambda_{i}\right)\left\|B_{i} T^{i-1} u_{n}-B_{i} T^{i-1} p\right\|^{2}\right\} \\
& \leq \frac{\alpha_{n} \rho \tau}{1+\alpha_{n}(\nu-\rho \tau)}\left\|x_{n}-p\right\|^{2}+\frac{2 \alpha_{n}}{1+\alpha_{n}(\nu-\rho \tau)}\left\langle\rho U(p)-\mu F(p), x_{n+1}-p\right\rangle \\
& \quad+\frac{\left(1-\alpha_{n} v\right) \beta_{n}}{1+\alpha_{n}(v-\rho \tau)}\left\|S x_{n}-p\right\|^{2}+\left\|x_{n}-p\right\|^{2}-\left\|x_{n+1}-p\right\|^{2} \\
& \leq \frac{\alpha_{n} \rho \tau}{1+\alpha_{n}(v-\rho \tau)}\left\|x_{n}-p\right\|^{2}
\end{aligned}
$$




$$
\begin{aligned}
& +\frac{2 \alpha_{n}}{1+\alpha_{n}(\nu-\rho \tau)}\left\langle\rho U(p)-\mu F(p), x_{n+1}-p\right\rangle \\
& +\frac{\left(1-\alpha_{n} \nu\right) \beta_{n}}{1+\alpha_{n}(\nu-\rho \tau)}\left\|S x_{n}-p\right\|^{2}+\left(\left\|x_{n}-p\right\|+\left\|x_{n+1}-p\right\|\right)\left\|x_{n}-x_{n+1}\right\| .
\end{aligned}
$$

Since $\lim _{n \rightarrow \infty} \alpha_{n}=0, \lim _{n \rightarrow \infty} \beta_{n}=0$ and $\lim _{n \rightarrow \infty}\left\|x_{n+1}-x_{n}\right\|=0$, we have

$$
\lim _{n \rightarrow \infty}\left\|B_{i} T^{i-1} u_{n}-B_{i} T^{i-1} p\right\|=0
$$

By Lemma 2.4, we obtain

$$
\begin{aligned}
\| y_{n} & -p \|^{2} \\
= & \left\|T^{N} u_{n}-T^{N} p\right\|^{2} \\
= & \left\|P_{C}\left(I-\lambda_{N} B_{N}\right) T^{N-1} u_{n}-P_{C}\left(I-\lambda_{N} B_{N}\right) T^{N-1} p\right\|^{2} \\
\leq & \left\langle\left(I-\lambda_{N} B_{N}\right) T^{N-1} u_{n}-\left(I-\lambda_{N} B_{N}\right) T^{N-1} p, T^{N} u_{n}-T^{N} p\right\rangle \\
= & \frac{1}{2}\left(\left\|y_{n}-p\right\|^{2}+\left\|\left(I-\lambda_{N} B_{N}\right) T^{N-1} u_{n}-\left(I-\lambda_{N} B_{N}\right) T^{N-1} p\right\|^{2}\right. \\
& \left.-\left\|\left(I-\lambda_{N} B_{N}\right) T^{N-1} u_{n}-\left(I-\lambda_{N} B_{N}\right) T^{N-1} p-\left(T^{N} u_{n}-T^{N} p\right)\right\|^{2}\right) \\
\leq & \frac{1}{2}\left(\left\|y_{n}-p\right\|^{2}+\left\|T^{N-1} u_{n}-T^{N-1} p\right\|^{2}\right. \\
& \left.-\left\|T^{N-1} u_{n}-T^{N} u_{n}+T^{N} p-T^{N-1} p-\lambda_{N}\left(B_{N} T^{N-1} u_{n}-B_{N} T^{N-1} p\right)\right\|^{2}\right),
\end{aligned}
$$

which implies

$$
\begin{aligned}
\| y_{n}- & p \|^{2} \\
\leq & \left\|T^{N-1} u_{n}-T^{N-1} p\right\|^{2} \\
& -\left\|T^{N-1} u_{n}-T^{N} u_{n}+T^{N} p-T^{N-1} p-\lambda_{N}\left(B_{N} T^{N-1} u_{n}-B_{N} T^{N-1} p\right)\right\|^{2} \\
= & \left\|T^{N-1} u_{n}-T^{N-1} p\right\|^{2}-\left\|T^{N-1} u_{n}-T^{N} u_{n}+T^{N} p-T^{N-1} p\right\|^{2} \\
& -\lambda_{N}^{2}\left\|B_{N} T^{N-1} u_{n}-B_{N} T^{N-1} p\right\|^{2} \\
& +2 \lambda_{N}\left\langle T^{N-1} u_{n}-T^{N} u_{n}+T^{N} p-T^{N-1} p, B_{N} T^{N-1} u_{n}-B_{N} T^{N-1} p\right\rangle \\
\leq & \left\|T^{N-1} u_{n}-T^{N-1} p\right\|^{2}-\left\|T^{N-1} u_{n}-T^{N} u_{n}+T^{N} p-T^{N-1} p\right\|^{2} \\
& +2 \lambda_{N}\left\|T^{N-1} u_{n}-T^{N} u_{n}+T^{N} p-T^{N-1} p\right\|\left\|B_{N} T^{N-1} u_{n}-B_{N} T^{N-1} p\right\| .
\end{aligned}
$$

By induction and (12), we have

$$
\begin{aligned}
\left\|y_{n}-p\right\|^{2} \leq & \left\|x_{n}-p\right\|^{2}-\sum_{i=1}^{N}\left\|T^{i-1} u_{n}-T^{i} u_{n}+T^{i} p-T^{i-1} p\right\|^{2} \\
& +\sum_{i=1}^{N} 2 \lambda_{i}\left\|T^{i-1} u_{n}-T^{i} u_{n}+T^{i} p-T^{i-1} p\right\|\left\|B_{i} T^{i-1} u_{n}-B_{i} T^{i-1} p\right\| .
\end{aligned}
$$


It follows from (21) and (34) that we have

$$
\begin{aligned}
& \left\|x_{n+1}-p\right\|^{2} \\
& \leq \frac{\alpha_{n} \rho \tau}{1+\alpha_{n}(\nu-\rho \tau)}\left\|x_{n}-p\right\|^{2}+\frac{2 \alpha_{n}}{1+\alpha_{n}(v-\rho \tau)}\left\langle\rho U(p)-\mu F(p), x_{n+1}-p\right\rangle \\
& +\frac{\left(1-\alpha_{n} v\right) \beta_{n}}{1+\alpha_{n}(v-\rho \tau)}\left\|S x_{n}-p\right\|^{2}+\frac{\left(1-\alpha_{n} v\right)\left(1-\beta_{n}\right)}{1+\alpha_{n}(\nu-\rho \tau)}\left\{\left\|x_{n}-p\right\|^{2}\right. \\
& -\sum_{i=1}^{N}\left\|T^{i-1} u_{n}-T^{i} u_{n}+T^{i} p-T^{i-1} p\right\|^{2} \\
& \left.+\sum_{i=1}^{N} 2 \lambda_{i}\left\|T^{i-1} u_{n}-T^{i} u_{n}+T^{i} p-T^{i-1} p\right\|\left\|B_{i} T^{i-1} u_{n}-B_{i} T^{i-1} p\right\|\right\} \\
& \leq \frac{\alpha_{n} \rho \tau}{1+\alpha_{n}(v-\rho \tau)}\left\|x_{n}-p\right\|^{2}+\frac{2 \alpha_{n}}{1+\alpha_{n}(v-\rho \tau)}\left\langle\rho U(p)-\mu F(p), x_{n+1}-p\right\rangle \\
& +\frac{\left(1-\alpha_{n} \nu\right) \beta_{n}}{1+\alpha_{n}(\nu-\rho \tau)}\left\|S x_{n}-p\right\|^{2}+\left\|x_{n}-p\right\|^{2} \\
& +\frac{\left(1-\alpha_{n} \nu\right)\left(1-\beta_{n}\right)}{1+\alpha_{n}(\nu-\rho \tau)}\left\{-\sum_{i=1}^{N}\left\|T^{i-1} u_{n}-T^{i} u_{n}+T^{i} p-T^{i-1} p\right\|^{2}\right. \\
& \left.+\sum_{i=1}^{N} 2 \lambda_{i}\left\|T^{i-1} u_{n}-T^{i} u_{n}+T^{i} p-T^{i-1} p\right\|\left\|B_{i} T^{i-1} u_{n}-B_{i} T^{i-1} p\right\|\right\},
\end{aligned}
$$

which implies

$$
\begin{aligned}
& \frac{\left(1-\alpha_{n} v\right)\left(1-\beta_{n}\right)}{1+\alpha_{n}(v-\rho \tau)}\left\{\sum_{i=1}^{N}\left\|T^{i-1} u_{n}-T^{i} u_{n}+T^{i} p-T^{i-1} p\right\|^{2}\right\} \\
& \leq \frac{\alpha_{n} \rho \tau}{1+\alpha_{n}(v-\rho \tau)}\left\|x_{n}-p\right\|^{2}+\frac{2 \alpha_{n}}{1+\alpha_{n}(v-\rho \tau)}\left\langle\rho U(p)-\mu F(p), x_{n+1}-p\right\rangle \\
& \quad+\frac{\left(1-\alpha_{n} v\right) \beta_{n}}{1+\alpha_{n}(v-\rho \tau)}\left\|S x_{n}-p\right\|^{2}+\left\|x_{n}-p\right\|^{2}-\left\|x_{n+1}-p\right\|^{2} \\
& \leq \frac{\alpha_{n} \rho \tau}{1+\alpha_{n}(v-\rho \tau)}\left\|x_{n}-p\right\|^{2}+\frac{2 \alpha_{n}}{1+\alpha_{n}(v-\rho \tau)}\left\langle\rho U(p)-\mu F(p), x_{n+1}-p\right\rangle \\
& \quad+\frac{\left(1-\alpha_{n} v\right) \beta_{n}}{1+\alpha_{n}(v-\rho \tau)}\left\|S x_{n}-p\right\|^{2}+\left(\left\|x_{n}-p\right\|+\left\|x_{n+1}-p\right\|\right)\left\|x_{n}-x_{n+1}\right\| \\
& \quad+\frac{\left(1-\alpha_{n} \nu\right)\left(1-\beta_{n}\right)}{1+\alpha_{n}(v-\rho \tau)}\left\{\sum_{i=1}^{N} 2 \lambda_{i}\left\|T^{i-1} u_{n}-T^{i} u_{n}+T^{i} p-T^{i-1} p\right\|\right. \\
& \left.\quad \times\left\|B_{i} T^{i-1} u_{n}-B_{i} T^{i-1} p\right\|\right\} .
\end{aligned}
$$

Since $\lim _{n \rightarrow \infty} \alpha_{n}=0, \lim _{n \rightarrow \infty} \beta_{n}=0$ and $\lim _{n \rightarrow \infty}\left\|B_{i} T^{i-1} u_{n}-B_{i} T^{i-1} p\right\|^{2}=0$, we have

$$
\lim _{n \rightarrow \infty}\left\|T^{i-1} u_{n}-T^{i} u_{n}+T^{i} p-T^{i-1} p\right\|=0
$$


From (37), we obtain

$$
\left\|u_{n}-y_{n}\right\|=\left\|T^{0} u_{n}-T^{N} u_{n}\right\| \leq \sum_{i=1}^{N}\left\|T^{i-1} u_{n}-T^{i} u_{n}+T^{i} p-T^{i-1} p\right\|,
$$

which means $\lim _{n \rightarrow \infty}\left\|u_{n}-y_{n}\right\|=0$. Note $\lim _{n \rightarrow \infty}\left\|u_{n}-x_{n}\right\|=0, \lim _{n \rightarrow \infty}\left\|u_{n}-y_{n}\right\|=0$, then we have $\lim _{n \rightarrow \infty}\left\|x_{n}-y_{n}\right\|=0$. Since $T\left(x_{n}\right) \in C_{1}$, we have

$$
\begin{aligned}
\left\|x_{n}-T\left(x_{n}\right)\right\| \leq & \left\|x_{n}-x_{n+1}\right\|+\left\|x_{n+1}-T\left(x_{n}\right)\right\| \\
& =\left\|x_{n}-x_{n+1}\right\|+\left\|P_{C_{1}}\left[V_{n}\right]-P_{C_{1}}\left[T\left(x_{n}\right)\right]\right\| \\
\leq & \left\|x_{n}-x_{n+1}\right\|+\| \alpha_{n}\left(\rho U\left(x_{n}\right)-\mu F\left(T\left(y_{n}\right)\right)+T\left(y_{n}\right)-T\left(x_{n}\right) \|\right. \\
\leq & \left\|x_{n}-x_{n+1}\right\|+\alpha_{n} \| \rho U\left(x_{n}\right)-\mu F\left(T\left(y_{n}\right)\|+\| y_{n}-x_{n} \|\right. \\
\leq & \left\|x_{n}-x_{n+1}\right\|+\alpha_{n} \| \rho U\left(x_{n}\right)-\mu F\left(T\left(y_{n}\right)\|+\| \beta_{n} S x_{n}+\left(1-\beta_{n}\right) y_{n}-x_{n} \|\right. \\
\leq & \left\|x_{n}-x_{n+1}\right\|+\alpha_{n} \| \rho U\left(x_{n}\right)-\mu F\left(T\left(y_{n}\right) \|\right. \\
& +\beta_{n}\left\|S x_{n}-x_{n}\right\|+\left(1-\beta_{n}\right)\left\|y_{n}-x_{n}\right\| .
\end{aligned}
$$

Noting that $\lim _{n \rightarrow \infty} \alpha_{n}=0, \lim _{n \rightarrow \infty} \beta_{n}=0, \lim _{n \rightarrow \infty}\left\|x_{n}-y_{n}\right\|=0$, and $\lim _{n \rightarrow \infty}\left\|x_{n+1}-x_{n}\right\|=$ 0 , we have $\lim _{n \rightarrow \infty}\left\|x_{n}-T\left(x_{n}\right)\right\|=0$.

Step 3. We show that $z \in F(T)$. Assume that $z \notin F(T)$. Since $x_{n_{i}}$ converges weakly to $z$ and $T z \neq z$, by Lemma 2.9, we have

$$
\begin{aligned}
& \liminf _{n \rightarrow \infty}\left\|x_{n_{i}}-z\right\| \\
& \quad<\liminf _{n \rightarrow \infty}\left\|x_{n_{i}}-T z\right\| \leq \liminf _{n \rightarrow \infty}\left(\left\|x_{n_{i}}-T x_{n_{i}}\right\|+\left\|T x_{n_{i}}-T z\right\|\right) \leq \liminf _{n \rightarrow \infty}\left\|x_{n_{i}}-z\right\|,
\end{aligned}
$$

which is a contradiction. Thus, we obtain $z \in F(T)$. To prove the convergence of the sequence $\left\{x_{n}\right\}$, we need to prove the following conclusion, that is, the sequence $\left\{x_{n}\right\}$ generated by (8) converges strongly to $w$, which is the unique solution of the variational inequality

$$
\langle\rho U(w)-\mu F(w), x-w\rangle \leq 0, \quad \forall x \in \Theta
$$

In fact, noting that $u_{n}=R_{r_{n, F_{1}}}\left(x_{n}+\gamma A^{*}\left(R_{r_{n, F_{2}}}-I\right) A x_{n}\right.$ and

$$
F_{1}\left(u_{n}, y\right)+\frac{1}{r_{n}}\left\langle y-u_{n}, u_{n}-x_{n}\right\rangle-\frac{1}{r_{n}}\left\langle y-u_{n}, \gamma A^{*}\left(R_{r_{n}, F_{2}}-I\right) A x_{n}\right\rangle \geq 0, \quad \forall y \in C_{1} .
$$

From the monotonicity of $F_{1}$, we have

$$
-\frac{1}{r_{n}}\left\langle y-u_{n}, \gamma A^{*}\left(R_{r_{n}, F_{2}}-I\right) A x_{n}\right\rangle+\frac{1}{r_{n}}\left\langle y-u_{n}, u_{n}-x_{n}\right\rangle \geq F_{1}\left(y, u_{n}\right), \quad \forall y \in C_{1},
$$

and

$$
-\frac{1}{r_{n_{i}}}\left\langle y-u_{n_{i}}, \gamma A^{*}\left(R_{r_{n_{i}}, F_{2}}-I\right) A x_{n_{i}}\right\rangle+\left\langle y-u_{n_{i}}, \frac{u_{n_{i}}-x_{n_{i}}}{r_{n_{i}}}\right\rangle \geq F_{1}\left(y, u_{n_{i}}\right), \quad \forall y \in C_{1} .
$$


Since $\left\|u_{n}-x_{n}\right\| \rightarrow 0,\left\|\left(R_{r_{n}, F_{2}}-I\right) A x_{n}\right\| \rightarrow 0$, we get $\left\{u_{n_{i}}\right\}$ converges weakly to $z$. By (A4), we know $F_{1}(y, z) \leq 0, \forall y \in C_{1}$. Let $y_{t}=t y+(1-t) z, t \in(0,1]$, it follows from $y \in C_{1}, z \in C_{1}$ and the convexity of $C_{1}$ that $F_{1}\left(y_{t}, z\right) \leq 0$. So, from (A1), (A3), and (A4), we have

$$
0=F_{1}\left(y_{t}, y_{t}\right) \leq t F_{1}\left(y_{t}, y\right)+(1-t) F_{1}\left(y_{t}, z\right) \leq F_{1}\left(y_{t}, y\right) .
$$

Therefore $F_{1}(z, y) \geq 0, \forall y \in C_{1}$. This is $z \in E P\left(F_{1}\right)$.

Next we show that $A z \in E P\left(F_{2}\right)$, since $\left\|u_{n}-x_{n}\right\| \rightarrow 0$, there exists a subsequence $\left\{x_{n_{k}}\right\}$ of $\left\{x_{n}\right\}$ such that $\left\{x_{n_{k}}\right\}$ converges weakly to $z$, and since $A$ is a bounded linear operator, $\left\{A x_{n_{k}}\right\}$ converges weakly to $A z$. Setting $\varpi_{n_{k}}=A x_{n_{k}}-R_{r_{n_{k}}, F_{2}} A x_{n_{k}}$, it follows tfrom $\lim _{n \rightarrow \infty} \|\left(R_{r_{n}, F_{2}}-\right.$ I) $A x_{n} \|=0$ that $\lim _{k \rightarrow \infty} \varpi_{n_{k}}=0$. By Lemma 2.1, we have

$$
F_{2}\left(A x_{n_{k}}-\varpi_{n_{k}}, y\right)+\frac{1}{r_{n_{k}}}\left\langle y-\left(A x_{n_{k}}-\varpi_{n_{k}}\right),\left(A x_{n_{k}}-\varpi_{n_{k}}\right)-A x_{n_{k}}\right\rangle \geq 0, \quad \forall y \in C_{2} .
$$

Since $F_{2}$ is upper semicontinuous in the first argument, taking limsup to the above inequality as $k \rightarrow \infty$, we have $F_{2}(A z, y) \geq 0, \forall y \in C_{2}$, which means that $A z \in E P\left(F_{2}\right)$, so $z \in \Gamma$. Next, we claim that $z \in \operatorname{Fix}(G)$. From Lemma 2.6, we know $G=T^{N}$ is nonexpansive, and

$$
\left\|y_{n}-G y_{n}\right\|=\left\|T^{N} u_{n}-T^{N} y_{n}\right\| \leq\left\|u_{n}-y_{n}\right\| .
$$

It follows from $\lim _{n \rightarrow \infty}\left\|u_{n}-x_{n}\right\|=0$ and $\lim _{n \rightarrow \infty}\left\|x_{n}-y_{n}\right\|=0$ that $\lim _{n \rightarrow \infty}\left\|y_{n}-G y_{n}\right\|=$ 0 . Furthermore, we get

$$
\begin{aligned}
\left\|x_{n}-G x_{n}\right\| \leq\left\|x_{n}-y_{n}\right\| & +\left\|y_{n}-G y_{n}\right\|+\left\|G y_{n}-G x_{n}\right\| \\
& \leq 2\left\|x_{n}-y_{n}\right\|+\left\|y_{n}-G y_{n}\right\|,
\end{aligned}
$$

which implies $\lim _{n \rightarrow \infty}\left\|x_{n}-G x_{n}\right\|=0$. Then, by Lemma 2.10, we obtain $z \in \operatorname{Fix}(G)$. Thus, we have $z \in \Theta$. Observe that the constants satisfy $0 \leq \rho \tau<v$ and $k \geq \eta$, from Lemma 2.7, the operator $\mu F-\rho U$ is $\mu \eta-\rho \tau$ strongly monotone. Then we get the uniqueness of the solution of the variational inequality and denote it by $w \in \Theta$.

Last, we show that $x_{n} \rightarrow w$. Note that

$$
\begin{aligned}
& \limsup _{n \rightarrow \infty}\left\langle\rho U(w)-\mu F(w), x_{n}-w\right\rangle \\
& \quad=\limsup _{i \rightarrow \infty}\left\langle\rho U(w)-\mu F(w), x_{n_{i}}-w\right\rangle=\langle\rho U(w)-\mu F(w), z-w\rangle \leq 0,
\end{aligned}
$$

and

$$
\begin{aligned}
\left\|x_{n+1}-w\right\|^{2} \\
\quad=\left\langle P_{C}\left[V_{n}\right]-w, x_{n+1}-w\right\rangle \\
\quad=\left\langle P_{C}\left[V_{n}\right]-V_{n}, P_{C}\left[V_{n}\right]-w\right\rangle+\left\langle V_{n}-w, x_{n+1}-w\right\rangle \\
\quad \leq\left\langle\alpha_{n}\left(\rho U\left(x_{n}\right)-\mu F(w)\right)+\left(I-\alpha_{n} \mu F\right)\left(T\left(z_{n}\right)\right)-\left(I-\alpha_{n} \mu F\right)(T(w)), x_{n+1}-w\right\rangle \\
\quad=\left\langle\alpha_{n} \rho\left(U\left(x_{n}\right)-U(w)\right), x_{n+1}-w\right\rangle+\alpha_{n}\left\langle\rho U(w)-\mu F(w), x_{n+1}-w\right\rangle
\end{aligned}
$$




$$
\begin{aligned}
& +\left\langle\left(I-\alpha_{n} \mu F\right)\left(T\left(z_{n}\right)\right)-\left(I-\alpha_{n} \mu F\right)(T(w)), x_{n+1}-w\right\rangle \\
\leq & \alpha_{n} \rho \tau\left\|x_{n}-w\right\|\left\|x_{n+1}-w\right\|+\alpha_{n}\left\langle\rho U(w)-\mu F(w), x_{n+1}-w\right\rangle \\
& +\left(1-\alpha_{n} v\right)\left\|z_{n}-w\right\|\left\|x_{n+1}-w\right\| \\
\leq & \alpha_{n} \rho \tau\left\|x_{n}-w\right\|\left\|x_{n+1}-w\right\|+\alpha_{n}\left\langle\rho U(w)-\mu F(w), x_{n+1}-w\right\rangle \\
& +\left(1-\alpha_{n} v\right)\left\{\beta_{n}\left\|S x_{n}-S w\right\|+\beta_{n}\|S w-w\|+\left(1-\beta_{n}\right)\left\|y_{n}-w\right\|\right\}\left\|x_{n+1}-w\right\| \\
\leq & \alpha_{n} \rho \tau\left\|x_{n}-w\right\|\left\|x_{n+1}-w\right\|+\alpha_{n}\left\langle\rho U(w)-\mu F(w), x_{n+1}-w\right\rangle \\
& +\left(1-\alpha_{n} v\right)\left\{\beta_{n}\left\|x_{n}-w\right\|+\beta_{n}\|S w-w\|+\left(1-\beta_{n}\right)\left\|x_{n}-w\right\|\right\}\left\|x_{n+1}-w\right\| \\
= & \left(1-\alpha_{n}(v-\rho \tau)\right)\left\|x_{n}-w\right\|\left\|x_{n+1}-w\right\|+\alpha_{n}\left\langle\rho U(w)-\mu F(w), x_{n+1}-w\right\rangle \\
& +\left(1-\alpha_{n} v\right) \beta_{n}\|S w-w\|\left\|x_{n+1}-w\right\| \\
\leq & \frac{\left(1-\alpha_{n}(v-\rho \tau)\right)}{2}\left(\left\|x_{n}-w\right\|^{2}+\left\|x_{n+1}-w\right\|^{2}\right)+\alpha_{n}\left\langle\rho U(w)-\mu F(w), x_{n+1}-w\right\rangle \\
& +\left(1-\alpha_{n} v\right) \beta_{n}\|S w-w\|\left\|x_{n+1}-w\right\|,
\end{aligned}
$$

which implies that

$$
\begin{aligned}
\left\|x_{n+1}-w\right\|^{2} \leq & \frac{1-\alpha_{n}(v-\rho \tau)}{1+\alpha_{n}(v-\rho \tau)}\left\|x_{n}-w\right\|^{2}+\frac{2 \alpha_{n}}{1+\alpha_{n}(v-\rho \tau)}\left\langle\rho U(w)-\mu F(w), x_{n+1}-w\right\rangle \\
& +\frac{2\left(1-\alpha_{n} \nu\right) \beta_{n}}{1+\alpha_{n}(v-\rho \tau)}\|S w-w\|\left\|x_{n+1}-w\right\| \\
\leq & \left(1-\alpha_{n}(v-\rho \tau)\right)\left\|x_{n}-w\right\|^{2} \\
& +\frac{2 \alpha_{n}(v-\rho \tau)}{1+\alpha_{n}(v-\rho \tau)}\left\{\frac{1}{v-\rho \tau}\left\langle\rho U(w)-\mu F(w), x_{n+1}-w\right\rangle\right. \\
& \left.+\frac{\left(1-\alpha_{n} v\right) \beta_{n}}{\alpha_{n}(v-\rho \tau)}\|S w-w\|\left\|x_{n+1}-w\right\|\right\} .
\end{aligned}
$$

Let $\sigma_{n}=\left\|x_{n}-w\right\|^{2}, \phi_{n}=\alpha_{n}(\nu-\rho \tau)$ and

$$
\begin{aligned}
\varphi_{n}= & \frac{2 \alpha_{n}(v-\rho \tau)}{1+\alpha_{n}(v-\rho \tau)}\left\{\frac{1}{v-\rho \tau}\left\langle\rho U(w)-\mu F(w), x_{n+1}-w\right\rangle\right. \\
& \left.+\frac{\left(1-\alpha_{n} v\right) \beta_{n}}{\alpha_{n}(v-\rho \tau)}\|S w-w\|\left\|x_{n+1}-w\right\|\right\} .
\end{aligned}
$$

Then the above inequality turns into the following:

$$
\sigma_{n+1}=\left(1-\phi_{n}\right) \sigma_{n}+\varphi_{n}
$$

From Conditions (i) and (ii) of Theorem 3.1, we have

$$
\begin{aligned}
& \phi_{n} \rightarrow 0(n \rightarrow \infty) \text { and } \\
& \limsup _{n \rightarrow \infty} \frac{\varphi_{n}}{\phi_{n}}=\limsup _{n \rightarrow \infty} \frac{2}{1+\alpha_{n}(v-\rho \tau)}\left\{\frac{1}{v-\rho \tau}\left\langle\rho U(w)-\mu F(w), x_{n+1}-w\right\rangle\right. \\
& \left.+\frac{\left(1-\alpha_{n} \nu\right) \beta_{n}}{\alpha_{n}(\nu-\rho \tau)}\|S w-w\|\left\|x_{n+1}-w\right\|\right\} \leq 0 \text {. }
\end{aligned}
$$


Then all conditions in Lemma 2.3 are satisfied, thus we can get $\sigma_{n} \rightarrow 0(n \rightarrow \infty)$, that is, $x_{n} \rightarrow w(n \rightarrow \infty)$. This completes the proof.

Corollary 3.1 For $i \in\{1,2\}$, let $H_{i}$ be a real Hilbert space, $C_{i}$ be a nonempty closed convex subset of $H_{i}$, let $F_{i}: C_{i} \times C_{i} \rightarrow R$ be an equilibrium function. Let $A: H_{1} \rightarrow H_{2}$ be bounded linear operators with their adjoint operators $A^{*}$. Let $B_{1}$ be $\xi_{1}$-inverse-strongly monotone. Let $F: C_{1} \rightarrow C_{1}$ be a k-Lipschitzian mapping and be $\eta$-strongly monotone, and let $U: C_{1} \rightarrow C_{1}$ be a $\tau$-Lipschitzian mapping. Let $S, T: C_{1} \rightarrow C_{1}$ be two nonexpansive mappings such that $\Theta=\Gamma \cap \operatorname{Fix}(G) \cap \operatorname{Fix}(T) \neq \emptyset$. For given $x_{0} \in C_{1}$ arbitrarily, let the iterative sequences $\left\{u_{n}\right\}$, $\left\{y_{n}\right\}$, and $\left\{x_{n}\right\}$ be generated by

$$
\left\{\begin{array}{l}
u_{n}=R_{r_{n}, F_{1}}\left(x_{n}+\gamma A^{*}\left(R_{r_{n}, F_{2}}-I\right) A x_{n}\right), \\
y_{n}=P_{C_{1}}\left(I-\lambda_{1} B_{1}\right) u_{n}, \\
z_{n}=\beta_{n} S x_{n}+\left(1-\beta_{n}\right) y_{n}, \\
x_{n+1}=P_{C_{1}}\left[\alpha_{n} \rho U\left(x_{n}\right)+\left(I-\alpha_{n} \mu F\right)\left(T\left(z_{n}\right)\right)\right],
\end{array}\right.
$$

where $\left\{r_{n}\right\} \subset(0, \infty), \gamma \in\left(0,1 / L_{A}\right), L_{A}$ is the spectral radius of the operators $A^{*} A$. Suppose that the parameters satisfy $0<\mu<\frac{2 \eta}{k^{2}}, k \geq \eta, 0 \leq \rho \tau<v$, where $v=1-\sqrt{1-\mu(2 \eta-\mu k)^{2}}$, and $\left\{\alpha_{n}\right\},\left\{\beta_{n}\right\}$ are the sequences in $(0,1)$ satisfying the following conditions:

(i) $\lim _{n \rightarrow \infty} \alpha_{n}=0$ and $\sum_{n=0}^{\infty} \alpha_{n}=\infty, \sum_{n=1}^{\infty}\left|\alpha_{n-1}-\alpha_{n}\right|<\infty$;

(ii) $\limsup _{n \rightarrow \infty} \frac{\beta_{n}}{\alpha_{n}}=0$, and $\beta_{n} \leq \alpha_{n}(n \geq 1), \sum_{n=1}^{\infty}\left|\beta_{n-1}-\beta_{n}\right|<\infty$;

(iii) $\liminf _{n \rightarrow \infty} r_{n}>0, \sum_{n=1}^{\infty}\left|r_{n-1}-r_{n}\right|<\infty$.

Then the sequence $\left\{x_{n}\right\}$ generated by (39) converges strongly to $w \in \Theta$.

Proof Putting $N=1$ in Theorem 3.1, we can conclude the desired conclusion directly.

\section{Conclusion}

In this paper, we considered a hierarchical fixed point problem (2), a split equilibrium problem (4)-(5), and a system of variational inequalities (7) in Hilbert spaces. An iterative algorithm for finding the common element of the solution sets of the three kinds of problems is presented. Strong convergence of the proposed algorithm is proved. The results presented here are new and very interesting.

Acknowledgements

The authors would like to thank the reviewers for their valuable comments, which have helped to improve the quality of this paper.

Funding

This research was supported by the National Natural Science Foundation of China, Liaoning Provincial Department of Education, and Liaoning Natural Fund Guidance Plan under project No. 11371070, No. LJ2019011, No. $2019-Z D-0502$.

Availability of data and materials

Not applicable.

Competing interests

The authors declare that they have no competing interests. 


\section{Publisher's Note}

Springer Nature remains neutral with regard to jurisdictional claims in published maps and institutional affiliations.

\section{Received: 4 March 2021 Accepted: 10 June 2021 Published online: 26 June 2021}

\section{References}

1. Anh, P.N., Anh, T.T.H., Hien, N.D.: Modified basic projection methods for a class of equilibrium problems. Numer. Algorithms 79, 139-152 (2018)

2. Bigi, G., Castellani, M., Pappalardo, M., Passacantando, M.: Nonlinear Programming Techniques for Equilibria. Springer, Switzerland (2019)

3. Blum, E., Oettli, W.: From optimization and variational inequalities to equilibrium problems. Math. Stud. $63,123-145$ (1994)

4. Browder, F.E.: Nonlinear Operators and Nonlinear Equations of Evolution in Banach Spaces. Am. Math. Soc., Washington (1976)

5. Byrne, C.: Iterative oblique projection onto convex sets and the split feasibility problem. Inverse Probl. 18, 441-453 (2002)

6. Byrne, $\mathrm{C}$ : A unified treatment of some iterative algorithms in signal processing and image reconstruction. Inverse Probl. 20, 103-120 (2004)

7. Cai, G., Bu, S.: Hybrid algorithm for generalized mixed equilibrium problems and variational inequality problems and fixed point problems. Comput. Math. Appl. 62, 4772-4782 (2011)

8. Ceng, L.C., Anasri, Q.H., Yao, J.C.: Some iterative methods for finding fixed points and for solving constrained convex minimization problems. Nonlinear Anal. 74, 5286-5302 (2011)

9. Ceng, L.C., Petrusel, A., Yao, J.C., Yao, Y.: Hybrid viscosity extragradient method for systems of variational inequalities, fixed points of nonexpansive mappings, zero points of accretive operators in Banach spaces. Fixed Point Theory 19, 487-502 (2018)

10. Ceng, L.C., Petrusel, A., Yao, J.C., Yao, Y.: Systems of variational inequalities with hierarchical variational inequality constraints for Lipschitzian pseudocontractions. Fixed Point Theory 20, 113-133 (2019)

11. Ceng, L.C., Wang, C.Y., Yao, J.C.: Strong convergence theorems by a relaxed extragradient method for a general system of variational inequalities. Math. Methods Oper. Res. 67, 375-390 (2008)

12. Censor, Y., Bortfeld, T., Martin, B., Trofimov, A.: Unified approach for inversion problems in intensity modulated radiation therapy. Phys. Med. Biol. 51, 2353-2365 (2006)

13. Censor, Y., Elfving, T.: A multiprojection algorithm using Bregman projection in product space. Numer. Algorithms 8 , 221-239 (1994)

14. Combettes, P.L.: The convex feasibility problem in image recovery. Adv. Imaging Electron Phys. 95, 155-453 (1996)

15. Combettes, P.L., Hirstoaga, S.A.: Equilibrium programming using proximal-like algorithms. Math. Program. 78, $117-136(1997)$

16. Combettes, P.L., Hirstoaga, S.A.: Equilibrium programming in Hilbert spaces. J. Nonlinear Convex Anal. 6, 117-136 (2005)

17. Dadashi, V., Postolache, M.: Hybrid proximal point algorithm and applications to equilibrium problems and convex programming. J. Optim. Theory Appl. 174(2), 518-529 (2017)

18. Dadashi, V., Postolache, M.: Forward-backward splitting algorithm for fixed point problems and zeros of the sum of monotone operators. Arab. J. Math. 9(1), 89-99 (2020)

19. Jitsupa, D., Juan, M.M., Kanokwan, S., Poom, K.: Convergence analysis of hybrid projection with CesÃ ro mean method for the split equilibrium and general system of finite variational inequalities. J. Comput. Appl. Math. 318, 658-673 (2017)

20. Konnov, L.V.: Equilibrium Models and Variational Inequalities. Elsevier, Amsterdam (2007)

21. Kzami, K.R., Rizvi, S.H.: Iterative approximation of a common solution of a split equilibrium problem, a variationa inequality problem and a fixed point problem. J. Egypt. Math. Soc. 21, 44-51 (2013)

22. Moudafi, A.: Krasnoselski Mann iteration for hierarchical fixed point problems. Inverse Probl. 23, 1635-1640 (2007)

23. Moudafi, A., Mainge, P.E.: Towards viscosity approximations of hierarchical fixed point problems. Fixed Point Theory Appl. 2006, Article ID 95453 (2006)

24. Opial, Z: Weak convergence of successive approximations for nonexpansive mappings. Bull. Am. Math. Soc. 73, 591-597 (1967)

25. Sahu, D.R., Pitea, A., Verma, M.: A new iteration technique for nonlinear operators as concerns convex programming and feasibility problems. Numer. Algorithms 83(2), 421-449 (2020)

26. Suzuki, T.: Moudafi's viscosity approximations with Meir-Keeler contractions. J. Math. Anal. Appl. 325, 342-352 (2007)

27. Thakur, B.S., Thakur, D., Postolache, M.: A new iterative scheme for numerical reckoning fixed points of Suzuki's generalized nonexpansive mappings. Appl. Math. Comput. 275, 147-155 (2016)

28. Thakur, B.S., Thakur, D., Postolache, M.: A new iteration scheme for approximating fixed points of nonexpansive mappings. Filomat 30(10), 2711-2720 (2016)

29. Usurelu, G.I., Bejenaru, A., Postolache, M.: Operators with property (E) as concerns numerical analysis and visualization. Numer. Funct. Anal. Optim. 41(11), 1398-1411 (2020)

30. Usurelu, G.I., Postolache, M.: Convergence analysis for a three-step Thakur iteration for Suzuki-type nonexpansive mappings with visualization. Symmetry 11(12), Article ID 1441 (2019)

31. Xu, H.K.: Viscosity approximation method for nonexpansive mappings. J. Math. Anal. Appl. 298(1), 279-291 (2004)

32. Xu, H.K.: Viscosity approximation method for nonexpansive mappings. J. Math. Anal. Appl. 298, 279-291 (2004)

33. Yao, Y., Agarwal, R.P., Postolache, M., Liu, Y.C.: Algorithms with strong convergence for the split common solution of the feasibility problem and fixed point problem. Fixed Point Theory Appl. 2014, Article ID 183 (2014)

34. Yao, Y., Li, H., Postolache, M.: Iterative algorithms for split equilibrium problems of monotone operators and fixed point problems of pseudo-contractions. Optimization (2020). https://doi.org/10.1080/02331934.2020.1857757

35. Yao, Y., Liou, Y.C., Yao, J.C.: Iterative algorithms for the split variational inequality and fixed point problems under nonlinear transformations. J. Nonlinear Sci. Appl. 10, 843-854 (2017) 
36. Yao, Y., Postolache, M., Yao, J.C.: Iterative algorithms for generalized variational inequalities. U.P.B. Sci. Bull., Series A. 81, 3-16 (2019)

37. Yao, Y., Postolache, M., Yao, J.C.: An iterative algorithm for solving generalized variational inequalities and fixed points problems. Mathematics 7, 61 (2019). https://doi.org/10.3390/math7010061

38. Yao, Y., Postolache, M., Yao, J.C.: Strong convergence of an extragradient algorithm for variational inequality and fixed point problems. U.P.B. Sci. Bull., Ser. A 82(1), 3-12 (2020)

39. Yao, Y.H., Cho, Y.J., Liou, Y.C.. Iterative algorithms for hierarchical fixed points problems and variational inequalities. Math. Comput. Model. 52(9-10), 1697-1705 (2010)

Submit your manuscript to a SpringerOpen ${ }^{\circ}$ journal and benefit from:

- Convenient online submission

- Rigorous peer review

- Open access: articles freely available online

- High visibility within the field

- Retaining the copyright to your article

Submit your next manuscript at $\boldsymbol{~ s p r i n g e r o p e n . c o m ~}$ 